\title{
Rheological Scaling of Ionic-Liquid-Based Polyelectrolytes in Ionic Liquid Solutions
}

\author{
Atsushi Matsumoto, ${ }^{* \dagger}$, $^{\circ}$ Francesco Del Giudice, ${ }^{\dagger, \ddagger}$ Rachapun Rotrattanadumrong, \\ and Amy Q. Shen* ${ }^{\dagger}+$ (0) \\ ${ }^{\dagger}$ Micro/Bio/Nanofluidics Unit, Okinawa Institute of Science and Technology Graduate University, 1919-1 Tancha, Onna-son, \\ Kunigami-gun, Okinawa 904-0495, Japan \\ ${ }^{\ddagger}$ Systems and Process Engineering Centre, College of Engineering, Swansea University, Fabian Way, Sewansea SA1 8EN, U.K.
}

Supporting Information

ABSTRACT: Polymerized ionic liquids (PILs) are a special class of polyelectrolytes with ionic liquid (IL) species being covalently attached to the repeating unit. The rheological properties of PIL in IL solutions are strongly influenced by the electrostatic screening between IL and PIL chains. However, the effect of IL electrostatic screening on the rheology of PIL in IL solutions remains elusive. To address this challenging yet important question, we conduct detailed rheological characterization of a model system containing a PIL [PC ${ }_{4}$-TFSI: poly(1-butyl-3-

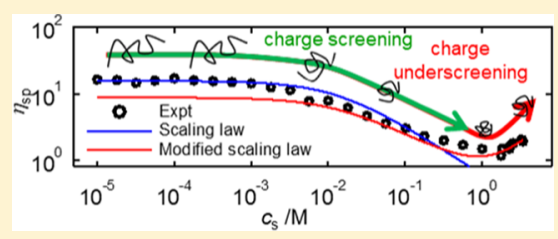
vinylimidazolium bis(trifluoromethanesulfonyl)imide)] in a mixture of a salt-free solvent (DMF: dimethylformamide) and an IL [Bmim-TFSI: 1-butyl-3-methylimidazolium bis(trifluoromethanesulfonyl)imide] solution, with low to high IL concentrations, while spanning dilute and semidilute polymer regimes. We compare the specific viscosity $\eta_{\mathrm{sp}}$ and the longest relaxation time $\lambda$ of PILs measured at various Bmim-TFSI concentrations from $0 \mathrm{M}$ (pure DMF) to $3.42 \mathrm{M}$ (pure Bmim-TFSI) with the scaling predictions for ordinary polyelectrolyte solutions. We find good agreement at low IL concentrations but significant deviations at higher IL concentrations. We capture this discrepancy by proposing and validating a modified scaling law accounting for the modified screening length in concentrated solutions of ordinary salts. We propose that extended PIL chains initially shrink due to the charge screening effect at low IL concentrations but revert to expanded configuration at higher IL concentrations due to the charge underscreening effect when the screening length increases with increasing IL concentrations. Our results shed new insights on the conformation of PIL in IL solutions and, for the first time, provide a valid reference for the study of general polyelectrolyte solutions at high salt concentrations, where the Debye-Hückel theory is no longer valid.

\section{INTRODUCTION}

Ionic liquids (ILs) are organic molten salts in the liquid state formed by relatively large cations or anions, weakly coordinated. ${ }^{1,2}$ Many ILs exist in the liquid state at room temperature due to their low melting temperatures. ILs are nonflammable with negligible vapor pressure, high ionic conductivity, and can absorb carbon dioxide with high selectivity and sensitivity. ${ }^{1,3-6}$ As a result, ILs have found applications as powerful solvents, ${ }^{7-10}$ catalysts for chemical reactions, ${ }^{11}$ and electrolytes for batteries. ${ }^{12}$

Polymerized ionic liquids (PILs) are a special class of polyelectrolytes with IL species being covalently attached to the repeating unit. ${ }^{2,13}$ Since PILs combine the unique features of ILs (electroconductivity and thermostability) with polymers (improved mechanical properties), they have been exploited to improve the performance of electrochemical devices and $\mathrm{CO}_{2}$ absorption membranes. ${ }^{14-17}$ The performance of PILs can be further enhanced by adding ILs in the solvent to increase the mobility of PIL chains. ${ }^{7-9,18}$ Within this context, the ability to control the viscoelastic properties of PILs is critical for PILbased material design and applications.

Existing studies show that electrostatic interactions play a predominant role in determining the viscoelastic properties of ordinary polyelectrolyte solutions. ${ }^{19-30}$ The strength of these interactions can be tuned by changing the amount of salt in the polyelectrolyte solution to regulate the charge screening effect. In addition, the viscoelastic properties of polymer solutions are related to the conformation of polymers in solutions. ${ }^{31}$ By coupling the scaling concepts in polymer physics ${ }^{32}$ with the Debye-Hückel theory, ${ }^{33}$ Dobrynin et al. ${ }^{19}$ proposed the scaling laws to correlate the viscoelastic properties with the conformation change of polyelectrolytes due to the charge screening effect, demonstrating good agreement for ordinary polyelectrolyte solutions. ${ }^{25,26,34-36}$ However, the scaling of Dobrynin et al. ${ }^{19}$ is based on the Debye-Hückel theory, ${ }^{33}$ which is valid only at relatively low salt concentrations. In fact, several research groups recently reported an unexpected long screening length in concentrated salt solutions (including both ionic liquids and ordinary salts), based on the measurements from the surface force apparatus. ${ }^{37-45}$ Specifically, Lee et al. ${ }^{43}$ showed that in the low salt concentration regime, the screening length in salt solutions decreased with increasing salt concentrations, in good agreement with the Debye-Hückel prediction. However, by further increasing the salt concen-

Received: November 29, 2018

Revised: March 15, 2019

Published: March 28, 2019 
Scheme 1. Synthesis of $\mathrm{PC}_{4}$-TFSI (PIL) Using the Counterion Conversion Method Proposed by Mecerreyes et al. ${ }^{46}$

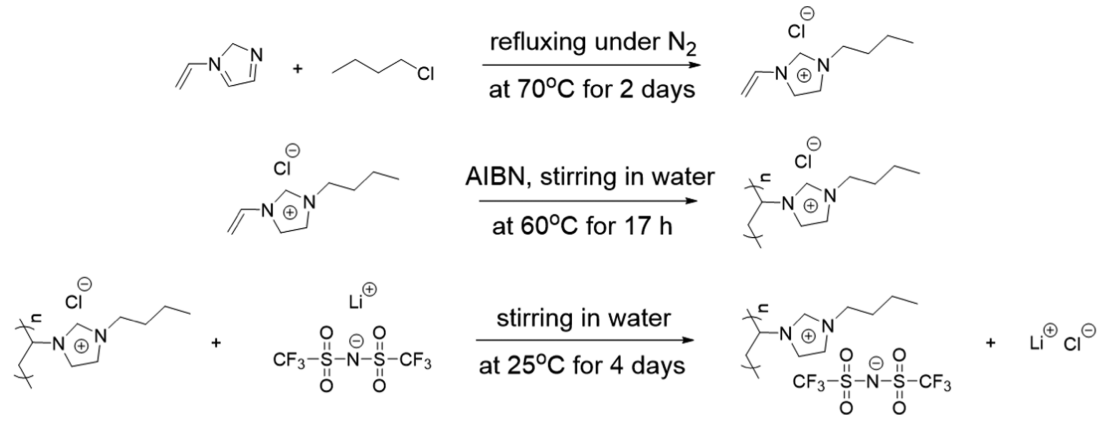

tration, the screening length started to increase again, a phenomenon denoted "charge underscreening". They also proposed a simple scaling relationship accounting for the modified screening length in concentrated salt solutions with respect to the Debye screening length. ${ }^{44}$

In this work, we wish to answer whether the viscoelastic properties of PILs in IL solutions can be captured by the scaling laws by Dobrynin et al. ${ }^{19}$ or by a modified scaling law involving the modified screening length described above. Specifically, we aim to understand the effect of the charge screening and underscreening on the viscoelastic properties and the conformation of PILs in IL solutions by investigating a polymerized ionic liquid, poly(1-butyl-3-vinylimidazolium bis(trifluoromethanesulfonyl)imide) ( $\mathrm{PC}_{4}$-TFSI), in a mixture of a salt-free solvent dimethylformamide (DMF) and an ionic liquid, 1-butyl-3-methylimidazolium bis (trifluoromethanesulfonyl)imide (Bmim-TFSI). We conducted systematic rheological characterizations to measure the specific viscosity $\eta_{\mathrm{sp}}$ and the longest relaxation time $\lambda$, by varying the molar concentration of Bmim-TFSI from salt-free to pure IL environment, spanning both dilute and semidilute polymer regimes, in both low and high salt environments. We compared our results with the scaling predictions of Dobrynin et al. ${ }^{19}$ for ordinary polyelectrolyte solutions. We found good agreement at low IL concentrations but significant deviations at higher IL concentrations. We explained this discrepancy by proposing and validating a modified scaling law, accounting for the modified screening length in concentrated salt solutions. Our results suggest that extended PIL chains initially shrink at low IL concentrations due to the charge screening effect observed in ordinary polyelectrolytes in salt solutions but become expanded again due to the charge underscreening effect at higher IL concentrations.

\section{EXPERIMENTAL SECTION}

2.1. Materials. 1-Vinylimidazole was purchased from Tokyo Chemical Industry, Japan, and used after distillation at $85^{\circ} \mathrm{C}$ under vacuum. Lithium bis(trifluoromethanesulfonyl)imide (Li-TFSI), 1chlorobutane, 2,2'-azobis(isobutyronitrile) (AIBN), superdehydrated dimethylformamide (DMF), and ethyl acetate were purchased from Wako Pure Chemicals, Japan. Silver nitrate $\left(\mathrm{AgNO}_{3}\right)$ at $0.1 \mathrm{M}$ in an aqueous solution was purchased from Sigma-Aldrich, Japan. 1-Butyl3-methylimidazolium bis(trifluoromethanesulfonyl)imide (BmimTFSI) (purity > 99.5\%) was purchased from Ionic Liquid Technologies, Germany. Deuterated water was purchased from Merck, Japan, and used as a solvent for NMR measurements. After passing through a Q-POD Element unit (Merck Millipore, Japan), Milli-Q water with a resistivity higher than $18.2 \mathrm{M} \Omega \mathrm{cm}$ was used as a solvent.

2.2. Synthesis of PILs. Scheme 1 summarizes the synthesis protocol of the PIL, poly(1-butyl-3-vinylimidazolium bis- (trifluoromethanesulfonyl)imide) $\left(\mathrm{PC}_{4}\right.$-TFSI $)$. The monomer 1-butyl-3-vinylimidazolium chloride $\left(\mathrm{C}_{4}-\mathrm{Cl}\right)$ was first prepared by quaternizing 1-vinylimidazole with 1-chlorobutane. After removing the unreacted 1-vinylimidazole and 1-chlorobutane, the polymerization of $\mathrm{C}_{4}-\mathrm{Cl}$ was carried out using AIBN as an initiator in Milli-Q water. Finally, $\mathrm{PC}_{4}$-TFSI was prepared using the counterion conversion method proposed by Mecerreyes et al. ${ }^{46}$ An inductively coupled plasma mass spectrometer (Element 2, Thermo Fisher Scientific Inc.) was used to identify the exchange rate to be almost $100 \%$ [mass fraction of residual ions $(\mathrm{g} / \mathrm{g}): \mathrm{Cl}^{-}=9.6 \times 10^{-4}$ and $\left.\mathrm{Li}^{+}=6.7 \times 10^{-5}\right]$. The details of the synthesis and the estimation of residual lithium $\left(\mathrm{Li}^{+}\right)$and chloride $\left(\mathrm{Cl}^{-}\right)$ion concentrations are provided in Sections S1 and S2 of the Supporting Information.

2.3. Molecular Weight of the Synthesized PILs. We estimated the molecular weight of the synthesized $\mathrm{PC}_{4}$-TFSI from the Zimm plot, ${ }^{47,48}$ obtained through static light scattering measurements on three dilute $\mathrm{PC}_{4}$-TFSI solutions with mass concentrations from 1.45 $\times 10^{-3}$ to $4.32 \times 10^{-3} \mathrm{~g} \mathrm{~cm}^{-3}$, which correspond to the monomer concentrations from $3.5 \times 10^{-3}$ to $1.0 \times 10^{-2} \mathrm{M}$ (Figure 1). At each mass concentration, the scattering intensity at a scattering angle ranging from 60 to $120^{\circ}$ with $10^{\circ}$ increment was measured using a light scattering spectrometer (3D LS spectrometer, LS Instruments). In our measurements, DMF mixed with $0.4 \mathrm{M}$ of Bmim-TFSI was chosen as the solvent to ensure that the measured mass concentrations were sufficiently high to allow the measurement of the intensity of the scattered light passing through $\mathrm{PC}_{4}$-TFSI solutions. By fitting the experimental data using the custom software provided by LS Instruments, the weight average molecular weight $M_{\mathrm{w}}$, the radius of gyration $R_{\mathrm{g}}$ and the second virial coefficient $A_{2}$ were estimated as $M_{\mathrm{w}}=(1.03 \pm 0.04) \times 10^{6} \mathrm{~g} \mathrm{~mol}^{-1}, R_{\mathrm{g}}=38 \pm 1.8 \mathrm{~nm}$, and $A_{2}=(2.5 \pm 0.8) \times 10^{-5} \mathrm{~mol} \mathrm{~mL} \mathrm{~g}$. The methods used to determine the optimized concentrations of $\mathrm{PC}_{4}$-TFSI and BmimTFSI and to estimate material properties $\left(M_{\mathrm{w}}, R_{\mathrm{g}}\right.$ and $\left.A_{2}\right)$ are provided in Sections S3.1 and S3.2 of the Supporting Information.

2.4. Preparation of the Test Mixtures. Test mixtures were prepared using two different methods, depending on the concentration of the PIL ( $\mathrm{PC}_{4}$-TFSI) and the IL (Bmim-TFSI). At low concentrations of $\mathrm{PC}_{4}$-TFSI and Bmim-TFSI, test mixtures were prepared by diluting concentrated $\mathrm{PC}_{4}$-TFSI and Bmim-TFSI solutions. At high concentrations of $\mathrm{PC}_{4}$-TFSI and Bmim-TFSI, test mixtures were prepared by directly adding the components into a glass vial. In both methods, $\mathrm{PC}_{4}$-TFSI quickly dissolved in individual solvents of DMF and Bmim-TFSI and the mixture of DMF and Bmim-TFSI at room temperature. These simple visual observations indicate that our solvent and solvent mixtures behave as good solvents for $\mathrm{PC}_{4}$-TFSI, further supported by the experimental result in which the measured specific viscosity follows the scaling prediction for ordinary polyelectrolytes in good solvents (see Section 3.1). The concentration of the ionic liquid Bmim-TFSI, $c_{\mathrm{s}}$, was calculated as $c_{\mathrm{s}}=m_{\mathrm{s}} \rho / M_{0} m_{\text {tot }}$ where $\rho$ and $M_{0}$ are the density of the mixture of DMF and Bmim-TFSI and the molar mass of Bmim-TFSI, whereas $m_{s}$ and $m_{\text {tot }}$ are the mass of Bmim-TFSI and the mass of the mixture of DMF and Bmim-TFSI, respectively. The value of $c_{\mathrm{s}}$ is varied from $0 \mathrm{M}$ (salt-free; pure DMF) to $3.42 \mathrm{M}$ (IL; pure Bmim-TFSI). The value of $\rho$ was measured using a density meter (DMA 35 Basic, Anton Paar) at 


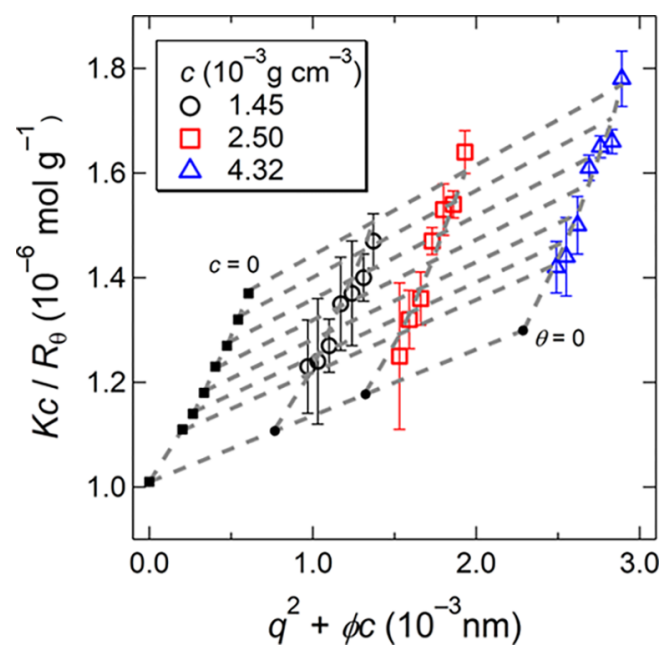

Figure 1. Zimm plot for dilute solutions of $\mathrm{PC}_{4}$-TFSI dissolved in DMF mixed with $0.4 \mathrm{M} \mathrm{Bmim-TFSI}$ at three different mass concentrations $c\left(\mathrm{~g} \mathrm{~cm}^{-3}\right): 1.45 \times 10^{-3}, 2.50 \times 10^{-3}$, and $4.32 \times$ $10^{-3}$ at $25^{\circ} \mathrm{C} . \mathrm{Kc} / R_{\theta}$ is plotted as a function of $\left(q^{2}+\phi c\right)$, where $K, R_{\theta}$, $q$, and $\phi$ are the optical constant, the excess Rayleigh ratio, the scattering vector calculated by $q \equiv \frac{4 \pi}{\lambda_{0}} \sin \frac{\theta}{2}$, and the shifting factor determined by LS Instruments software, respectively. Here, $\lambda_{0}$ $(632.8 \mathrm{~nm})$ is the wavelength of the incident light. $R_{\theta}$ at each mass concentration was measured at different scattering angles $\theta$ ranging from 60 to $120^{\circ}$. Dashed lines indicate the fitting curves used to extrapolate $K c / R_{\theta}$ to $c=0$ (filled squares) and $\theta=0$ (filled circles). The measured parameters are the molecular weight $M_{\mathrm{w}}=(1.03 \pm$ $0.04) \times 10^{6} \mathrm{~g} \mathrm{~mol}^{-1}$, the radius of gyration $R_{\mathrm{g}}=38 \pm 1.8 \mathrm{~nm}$, and the second virial coefficient $A_{2}=(2.5 \pm 0.8) \times 10^{-5} \mathrm{~mol} \mathrm{~mL} \mathrm{~g}{ }^{-2}$. The experimental error at each scattering angle and mass concentration was obtained from 10 independent measurements, whereas each material property and its corresponding standard deviation were obtained from three individual fittings.

room temperature $\left(\simeq 25{ }^{\circ} \mathrm{C}\right)$ (Figure S5). The monomer concentration of $\mathrm{PC}_{4}$-TFSI, $c_{\mathrm{p}}$, was calculated as a ratio of the number of moles of the repeating unit of $\mathrm{PC}_{4}$-TFSI to the volume of the mixture of DMF and Bmim-TFSI: $c_{\mathrm{p}}=m_{\mathrm{p}} \rho / M_{\mathrm{r}} m_{\text {tot }}$ where $m_{\mathrm{p}}$ is the mass of $\mathrm{PC}_{4}$-TFSI and $M_{\mathrm{r}}=431.43 \mathrm{~g} \mathrm{~mol}^{-1}$ is the molar mass of the repeating unit associated with the counteranion. Since DMF is a hygroscopic solvent, sample preparation was conducted in a glovebox under an argon atmosphere. The prepared solutions were hermetically sealed in borosilicate glass vials and then stored in a desiccator while maintaining humidity lower than $20 \%$ until use.

2.5. Effective Charges on $\mathrm{PC}_{4}$-TFSI Chains. Polyelectrolytes are polymers having ionic groups on their repeating units. These ionic groups dissociate in solutions, such as aqueous solutions, so that chains are ionized and counterions are dispersed in the solution. Manning ${ }^{49}$ has predicted theoretically that counterions can condense into polyelectrolyte chains as the number of ionic groups increases. Such ion condensation can be characterized by a quantity of $A$, representing the number of monomers between uncondensed charges (see more details from the scaling theory of Dobrynin et al. ${ }^{19}$ ). Thus, the value of $A$ for a polyelectrolyte in a given solvent is an important material parameter. Among reported techniques, ${ }^{19,26,50,51}$ we performed osmotic pressure measurements to determine $A$ for $\mathrm{PC}_{4^{-}}$ TFSI solutions. The osmotic pressure was measured using a commercially available membrane osmometer (Osmomat090, Gonotec, $\mathrm{GmbH}$.). We used a semipermeable membrane made of regenerated cellulose, designed for measurements using organic solvents, such as DMF. We experimentally confirmed that the membrane did not dissolve in Bmim-TFSI, consistent with the literature reports. ${ }^{10,52}$ The nominal molecular weight cutoff (MWCO) of the membrane, related to the pore size, was specified as MWCO =
$20000 \mathrm{Da}$ by the manufacturer. All of the measurements were performed at $25{ }^{\circ} \mathrm{C}$.

According to the scaling theory by Dobrynin et al., ${ }^{19}$ the osmotic pressure $\Pi$ for semidilute polyelectrolyte solutions stems from two contributions: $\Pi_{p}$ from polyelectrolyte chains and $\Pi_{i}$ from counterions and salt ions, given by

$$
\begin{aligned}
& \frac{\Pi_{\mathrm{p}}}{R T} \propto \frac{1}{\xi^{3}} \\
& \frac{\Pi_{\mathrm{i}}}{R T} \propto \frac{c_{\mathrm{p}}^{2}}{\left(4 A^{2} c_{\mathrm{s}}+A c_{\mathrm{p}}\right)}
\end{aligned}
$$

where $\xi$ is the correlation length of the polyelectrolyte chain. Since the polymer contribution $\Pi_{\mathrm{p}}$ is negligible, ${ }^{19} \Pi \approx \Pi_{\mathrm{i}}$. Thus, the value of $A$ can be estimated by fitting eq 2 with the measured $\Pi$.

In Figure 2, we plotted the measured $\Pi$ at $c_{\mathrm{p}}=4.0 \times 10^{-2} \mathrm{M}$ (open circles) as a function of the ratio of $c_{\mathrm{p}} / c_{\mathrm{s}}$. The value of $\Pi$ was initially a

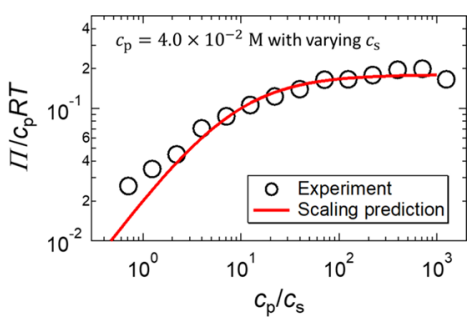

Figure 2. Osmotic pressure of semidilute $\mathrm{PC}_{4}$-TFSI solutions at $c_{\mathrm{p}}=$ $4.0 \times 10^{-2} \mathrm{M}$ as a function of $c_{\mathrm{s}}$. Open symbols represent the experimental data obtained by an osmometer (Osmomat090, Gonotec, GmbH.) at $25{ }^{\circ} \mathrm{C}$. The red curve represents the scaling prediction of $\Pi$ for semidilute flexible polyelectrolyte solutions (eq 2) with $A=2$. The error bars, estimated from three individual measurements, are smaller than the symbol size.

constant until $c_{\mathrm{s}}=1.0 \times 10^{-3} \mathrm{M}$, across which the value of $\Pi$ decreased with increasing $c_{s}$, i.e., with decreasing $c_{\mathrm{p}} / c_{\mathrm{s}}$, in good agreement with previous reports on ordinary polyelectrolyte solutions. ${ }^{19}$ Since all of the tested solutions fall under the semidilute regime with $c_{\mathrm{s}}$ ranging from $3.2 \times 10^{-5}$ to $5.6 \times 10^{-2} \mathrm{M}$ (further validation shown in Figure 5d), eq 2 is used to fit the measured $\Pi$ with $c_{\mathrm{p}} / c_{\mathrm{s}}$ (solid curve) with $A=2$ obtained as the best-fitting parameter.

We compared the estimated $A=2$ with the predicted $A$ by the Manning theory. ${ }^{49}$ When the charge density on a polyelectrolyte chain is large, such as $\mathrm{PC}_{4}$-TFSI, the value of $A$ can be estimated as the ratio of the Bjerrum length $l_{\mathrm{B}}=\frac{e^{2}}{4 \pi \varepsilon_{0} \mathrm{\epsilon}_{\mathrm{r}} k_{\mathrm{B}} T}$ to the monomer size $b$, i.e., $A=l_{\mathrm{B}} / b{ }^{49}$ Here, $\epsilon_{0}, \epsilon_{\mathrm{r}}, e$, and $k_{\mathrm{B}}$ are the dielectric constant of vacuum, the dielectric constant of a solvent, the elementary charge, and the Boltzmann constant, respectively. With $l_{\mathrm{B}}=1.5 \mathrm{~nm}$ in DMF $\left(\epsilon_{\mathrm{r}}=37\right)$ and $b=0.25 \mathrm{~nm}$ for vinyl polyelectrolytes, $A$ is estimated to be $A=6$, larger than the value $A=2$ obtained using the osmometer. We hypothesize that in our system weakly coordinated ionic liquid ions suppress the counterion condensation, which acts differently from the ordinary polyelectrolytes with strongly coordinated ions.

2.6. Shear Viscosity Measurements. The shear viscosity provides important information related to the molecular conformation and overlap and entanglement concentrations. In most of our studies, the polymer concentration is very low; thus, the measurement of the shear viscosity at low and high shear rates can be challenging. We employed several complementary rheological techniques, as described below.

2.6.1. Conventional Bulk Shear Rheometry. The shear viscosity $\eta$ was measured using a strain-controlled rheometer (ARES-G2, TA Instruments) at shear rates ranging from 0.1 to $1000 \mathrm{~s}^{-1}$. The measurements were performed with a stainless steel cone and plate geometry with $50 \mathrm{~mm}$ diameter and $1^{\circ}$ cone angle. The temperature 

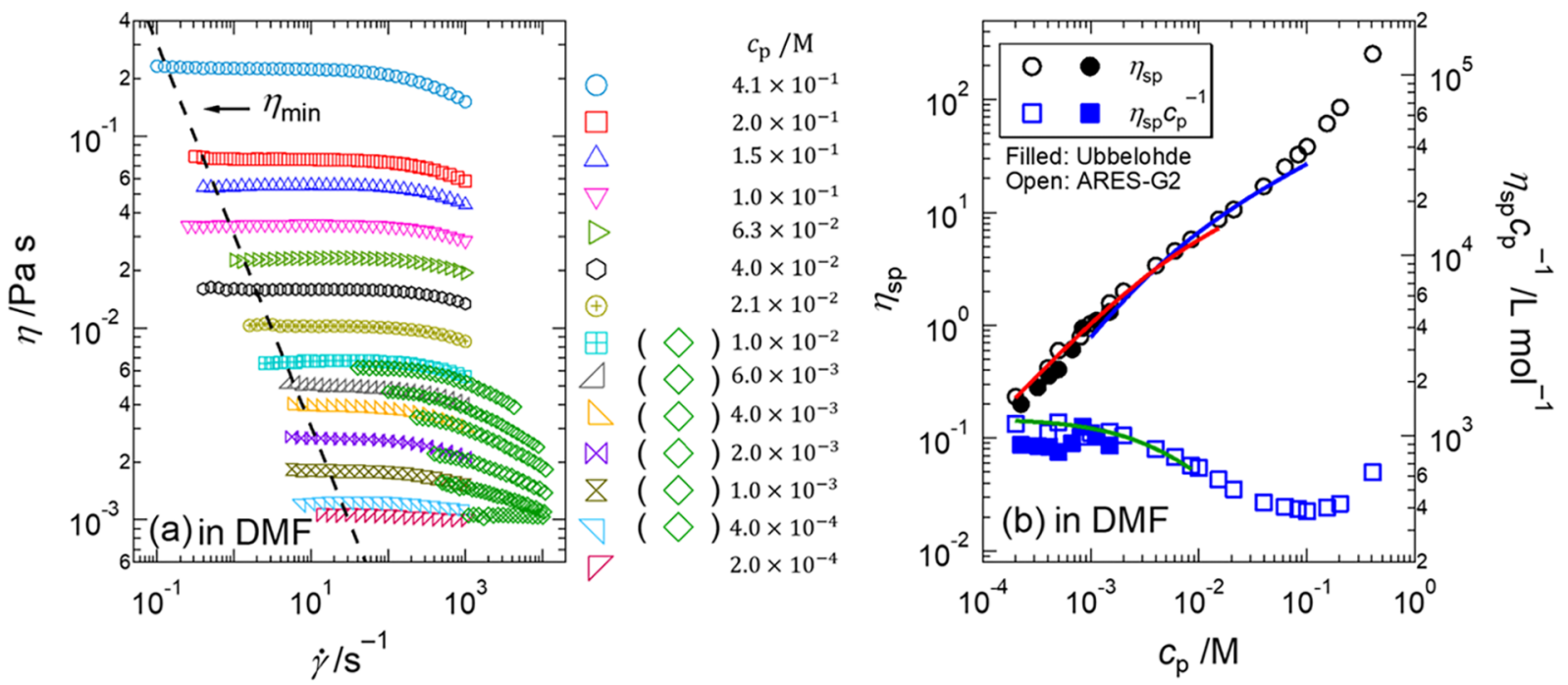

Figure 3. (a) Shear viscosity curves for $\mathrm{PC}_{4}$-TFSI solutions at $c_{\mathrm{s}}=0 \mathrm{M}$ [salt-free; pure dimethylformamide (DMF)] with different polymer concentrations measured using the microfluidic slit rheometer ( $\mathrm{m}$-VROC: open diamonds) and the strain-controlled rheometer (ARES-G2: open symbols with the other shapes) at $25{ }^{\circ} \mathrm{C}$. The dashed line indicates the minimum measurable shear viscosity for the strain-controlled rheometer equipped with a stainless steel cone and plate geometry with $50 \mathrm{~mm}$ diameter and $1^{\circ}$ cone angle (see Section S5.1 for more details). (b) Polymer concentration regimes of the synthesized $\mathrm{PC}_{4}$-TFSI at $c_{\mathrm{s}}=0 \mathrm{M}$ (DMF as the solvent) are determined from the power-law fit of the specific viscosity $\eta_{\mathrm{sp}}$ versus the monomer concentration $c_{\mathrm{p}}$ of PC $\mathrm{PC}_{4}$-TFSI. The values of $\eta_{\mathrm{sp}}$ [open (ARES-G2) and filled (Ubbelohde) circles] and the reduced viscosity $\left(\eta_{\mathrm{sp}} / c_{\mathrm{p}}\right)$ [open (ARES-G2) and filled (Ubbelohde) squares] are plotted as a function of $c_{\mathrm{p}}$. The red and blue curves are the fits to the experimental data of the scaling laws of $\eta_{\mathrm{sp}}$ for dilute (DF) and semidilute unentangled (SUF) solutions with $c_{\text {res }}=1.1 \mathrm{mM}$ and $A=2$. The green curve is the fit to the experimental data of $\left(\eta_{\text {sp }} / c_{\mathrm{p}}\right)$ for DF solutions with $c_{\text {res }}=1.1 \mathrm{mM}$ and $A=2$. The error bars are smaller than the symbol size. The scaling laws are summarized in Table 1 .

was set at $25{ }^{\circ} \mathrm{C}$ and controlled by an advanced Peltier system (TA Instruments) with a temperature accuracy of $\pm 0.1{ }^{\circ} \mathrm{C}$. The loaded solution was covered with a solvent trap to avoid the risk of both moisture absorption from the ambient environment and the sample evaporation.

Note that the shear viscosity generally decreases as the polymer concentration decreases; thus, caution is needed for the measurement at low polymer concentrations due to the sensitivity of the transducers on conventional rheometers (more detailed discussion in Section S5.1 of the Supporting Information).$^{53-55}$ To enhance the reliability of the viscosity measurement, high shear rates may be employed, where other features such as shear-thinning are also detectable. In cone and plate fixtures, high shear rates should be avoided due to the breakdown of measurement assumptions, such as steady, laminar, and isothermal flows and spherical liquid boundary condition to estimate the value of $\eta .^{53,56}$ Moreover, the radial migration effect, in which fluids are thrown out of the gap due to sufficiently large centrifugal forces, may take place at high shear rates. ${ }^{57}$ For these reasons, we compared the viscosity measured via the bulk rheometer (ARES-G2) with that from independent measurements carried out using a microfluidic-based rheometer and a gravity-driven capillary viscometer.

2.6.2. Microfluidic-Based Rheometry: the $m$-VROC. The shear viscosity $\eta$ at shear rates from 300 to $10000 \mathrm{~s}^{-1}$ was measured using a commercially available microfluidic slit rheometer (m-VROC, RheoSense Inc.). ${ }^{58}$ In our study, we employed a straight rectangular channel with $50 \mu \mathrm{m}$ in height, $3.0 \mathrm{~mm}$ in width, and $8.8 \mathrm{~mm}$ in length. This microfluidic configuration minimizes viscous heating and guarantees low inertia. ${ }^{58-61}$ The value of $\eta$ was estimated from the pressure detected through four microelectromechanical-system-based pressure transducers located along the centerline of the channel. A thermal jacket was used to maintain the temperature of test solutions and the channel at $25{ }^{\circ} \mathrm{C}$. The measurement principle is provided in the Supporting Information (Section S4.1).

2.6.3. Gravity-Driven Capillary Viscometry. An Ubbelohde viscometer (SHIBATA Science Technology Ltd.), a type of gravitydriven capillary viscometers, was also used to measure the specific viscosity $\eta_{\text {sp }}$ of test solutions at $25^{\circ} \mathrm{C}$. The Ubbelohde viscometer used in this study was designed to measure the kinematic viscosity $(=\eta / \rho)$ ranging from 0.3 to $1 \mathrm{~mm}^{2} \mathrm{~s}^{-1}$. Under the assumption of the Hagen-Poiseuille flow for incompressible Newtonian fluids, the value of $\eta_{\mathrm{sp}}$ can be estimated by ${ }^{56}$

$$
\eta_{\mathrm{sp}}=\frac{\rho_{\mathrm{p}} t_{\mathrm{p}}}{\rho_{\mathrm{s}} t_{\mathrm{s}}}-1
$$

where $\rho_{\mathrm{p}}$ and $t_{\mathrm{p}}$ are the density of a polymer solution and the time for the polymer solution to flow between two markings printed on the gravity-driven Ubbelohde viscometer, whereas $\rho_{\mathrm{s}}$ and $t_{\mathrm{s}}$ are the density of the solvent and the time for the solvent to flow between the same two markers at the same temperature, respectively. In our study, the times $t_{\mathrm{p}}$ and $t_{\mathrm{s}}$ were evaluated through a custom-made video tracking MATLAB code in which the meniscus position of the fluid in the viscometer was monitored (see Section S4.2 for more details). The temperature was maintained at $25{ }^{\circ} \mathrm{C}$ using a circulating water bath setup.

2.7. Measurement of the Longest Relaxation Times. The longest relaxation time $\lambda$ of a viscoelastic liquid is an important rheological parameter. However, when the polymer concentration is low, such measurement becomes even more challenging than the viscosity measurement. Here, we employed three rheological techniques: (i) conventional bulk shear rheology within the instrumentation limit, (ii) microrheology, and (iii) microfluidicbased rheometry, depending on the polymer concentration. The specific description is provided below.

2.7.1. Conventional Bulk Shear Rheometry. In conventional bulk shear rheometry, the relaxation time can be generally estimated through small-amplitude oscillatory shear measurements in the linear viscoelastic regime where the complex modulus is independent of the shear strain. ${ }^{56}$ The storage modulus $G^{\prime}$ and the loss modulus $G^{\prime \prime}$ in a frequency range of $0.1-100 \mathrm{rad} \mathrm{s}^{-1}$ were measured using a straincontrolled rheometer (ARES-G2, TA Instruments) with a stainless steel cone and plate geometry $\left(50 \mathrm{~mm}\right.$ in diameter and $1^{\circ}$ cone angle). The longest relaxation time was identified by the intersection of the slopes of $G^{\prime}$ and $G^{\prime \prime}$ at low frequencies (i.e., so-called terminal behavior). ${ }^{56}$ The temperature was kept at $25{ }^{\circ} \mathrm{C}$. 
2.7.2. Microrheology: Dynamic Light Scattering (DLS). Microrheology enables the measurement of $G^{\prime}$ and $G^{\prime \prime}$ in a wider range of frequency compared to the conventional rheometry. Among the microrheological techniques, we performed dynamic light scattering (DLS) experiments at $25{ }^{\circ} \mathrm{C}$, using a light scattering spectrometer (3D LS spectrometer, LS Instruments). This method is based on the analysis of the laser fluctuations generated by the collective Brownian motion of diluted particles suspended in the solution of interest. Silica particles with $0.5 \mu \mathrm{m}$ in diameter were suspended in $\mathrm{PC}_{4}$-TFSI solutions at $0.001 \mathrm{vol} \%$. The time-averaged autocorrelation function was measured at a scattering angle of $25^{\circ}$ with an averaging time of $1200 \mathrm{~s}$. The values of $G^{\prime}$ and $G^{\prime \prime}$ were derived using the method described by Mason et al., ${ }^{62,63}$ and the value of $\lambda$ was estimated as for conventional rheological techniques. More details can be found in the Supporting Information (Section S4.3).

2.7.3. Microfluidic-Based Rheometry: the $\mu$-Rheometer. Among the numerous "rheometry-on-a-chip" technologies, ${ }^{60,64}$ we employed the $\mu$-rheometer, ${ }^{65}$ which enables measurements of $\lambda$ down to microseconds, by observing the transverse migration of micrometerscale particles in a Poiseuille flow. Compared to other microfluidic techniques, the $\mu$-rheometer was successfully tested on several aqueous solutions of polyelectrolytes ${ }^{36,66}$ and on organic solutions containing polystyrene. ${ }^{67}$ In our study, we used a cylindrical glass capillary due to the chemical stability of glassware against DMF and Bmim-TFSI. Two channels with diameters $D$ of 100 and $50 \mu \mathrm{m}$ were employed according to the "sensitivity" of the measurements (smaller values of $D$ allow measurements of smaller $\lambda$; see eq 4).

For particles with diameter $d_{\mathrm{p}}$ flowing in a cylindrical channel with diameter $D$ at a volumetric flow rate $Q$ the longest relaxation time $\lambda$ can be estimated as ${ }^{36}$

$$
\lambda=2 \pi \frac{\pi}{4} \frac{1}{\beta^{2}} \frac{D^{4}}{L Q} \sqrt{\frac{1}{C} \ln \frac{f_{1} B}{1-f_{1}}}
$$

where $L$ is the distance from the channel inlet, $\beta=d_{\mathrm{p}} / D$ is the confinement ratio, $B=2.7$ and $C=2.75$ are the constants predicted by the theoretical model at $\beta \sim 0.1$, and $f_{1}$ is the normalized fraction of particles at the distance $L$. All measurements were performed at room temperature $\left(\simeq 25^{\circ} \mathrm{C}\right)$. The measurement principle is detailed in the Supporting Information (Section S4.4).

\section{RESULTS AND DISCUSSION}

3.1. Salt-Free Solutions: $\mathrm{PC}_{4}$-TFSI in DMF. 3.1.1. Overlap Concentration and Power-Law Regimes. To explore the polymeric nature of the polymerized ionic liquid, we first investigated the shear viscosity $\eta$ as a function of the shear rate $\dot{\gamma}$ for $\mathrm{PC}_{4}$-TFSI in pure DMF (salt-free), while varying the monomer concentration $c_{\mathrm{p}}$ of $\mathrm{PC}_{4}$-TFSI (Figure $3 \mathrm{a}$ ). The shear viscosity $\eta$ was measured using a microfluidic slit rheometer ( $\mathrm{m}$-VROC, open diamonds) and a strain-controlled rheometer (ARES-G2, open symbols with the other shapes). Only representative values of $c_{\mathrm{p}}$ are shown in Figure 3a for clarity. For a given $c_{\mathrm{p}}, \eta$ remained constant at low shear rates but started to decrease above a critical shear rate $\dot{\gamma}^{*}$. At low $c_{\mathrm{p}}, \dot{\gamma}^{*}$ decreased with increasing $c_{\mathrm{p}}$, followed by an increase of $\dot{\gamma}^{*}$ with increasing $c_{\mathrm{p}}$ at intermediate $c_{\mathrm{p}}$. By further increasing $c_{\mathrm{p}}$, the value of $\dot{\gamma}^{*}$ decreased again with increasing $c_{\mathrm{p}}$. Such trend of $\dot{\gamma}^{*}$ versus $c_{\mathrm{p}}$ is consistent with previous reports on ordinary saltfree polyelectrolyte solutions. ${ }^{25,26,28,34,36,66,68}$

Important rheological information, such as overlap concentration and solvent quality, can be derived from the specific viscosity $\eta_{\text {sp }}$ [see open (ARES-G2) and filled (Ubbelohde) circles in Figure $3 \mathrm{~b}]$. The values of $\eta_{\mathrm{sp}}$ measured by ARES-G2 were estimated by $\eta_{\mathrm{sp}}=\left(\eta_{0}-\eta_{\mathrm{s}}\right) / \eta_{\mathrm{s}}$, where $\eta_{0}$ is the zero-shear viscosity of the polymer solution and $\eta_{s}$ is the solvent viscosity (in this case pure DMF). The values of $\eta_{0}$ were derived from the data of Figure 3a at low shear rates. The values of $\eta_{\mathrm{sp}}$ measured by ARES-G2 were in good agreement with those measured by the Ubbelohde viscometer. We observed two clear distinct power-law regimes with respect to $c_{\mathrm{p}}$. The specific viscosity shows $\eta_{\mathrm{sp}} \propto c_{\mathrm{p}}^{1.0}$ when $c_{\mathrm{p}} \leq 10^{-3} \mathrm{M}$ but displays $\eta_{\mathrm{sp}} \propto c_{\mathrm{p}}^{0.8}$ when $10^{-3} \mathrm{M}<c_{\mathrm{p}} \leq 10^{-1} \mathrm{M}$. The transition between the two scalings occurred around $\eta_{\mathrm{sp}} \sim 1.0$, which generally marks the transition between the dilute polymer regime (DF, where hydrodynamic interactions are dominant) and semidilute unentangled polymer regime (SUF, where hydrodynamic interactions are fully screened). ${ }^{31}$ The transition between the two polymer regimes occurred at the overlap concentration $c^{*} \sim 10^{-3} \mathrm{M}$. Chen and Elabd ${ }^{69}$ reported that the specific viscosity for PIL salt-free solutions in the SUF regime scaled as $\eta_{\mathrm{sp}} \propto c_{\mathrm{p}}^{0.5}$. We anticipate that our apparent scaling of $\eta_{\mathrm{sp}} \propto c_{\mathrm{p}}^{0.8}$ in the SUF regime implies the presence of residual salts in DMF; see more details below.

3.1.2. Concentration of Residual Salts. The solvent quality can be derived from the scaling of the specific viscosity in the SUF regime for flexible polymers, $\eta_{\mathrm{sp}} \propto c^{1 /(3 \nu-1)}$, where $\nu$ is the dimensionless scaling exponent. ${ }^{20}$ Taking $\eta_{\mathrm{sp}} \propto c_{\mathrm{p}}^{0.8}$ shown in Figure $3 \mathrm{~b}, \nu$ can be extracted as $\nu=0.75$, which lies between the value for polyelectrolytes in a good salt-free solvent $(\nu=1)$ and that for neutral polymers in a good solvent $(\nu=0.588)$. This value suggests that the local conformation of $\mathrm{PC}_{4}$-TFSI in DMF lies between fully extended $(\nu=1)$ and fully coiled $(\nu=0.588)$ chains due to partial screening of the electrostatic interactions. This scenario corresponds to the presence of the "residual salt" in our system, as also observed in other polyelectrolyte systems reported in literature. ${ }^{26,28,36,70}$ The origin of the residual salt is still unknown, but it may be related to the contamination during solvent and polymer preparations or to the pollution from the external environment. A specific value of the concentration of residual salts $c_{\text {res }}$ can be derived from the scaling law of Dobrynin et al. ${ }^{19}$ for the specific viscosity $\eta_{\text {sp,SUF }}$ spanning the SUF regime in a salty environment

$$
\eta_{\mathrm{sp}, \mathrm{SUF}} \propto c_{\mathrm{p}}^{1 / 2}\left[1+\frac{2 A c_{\text {ion }}}{c_{\mathrm{p}}}\right]^{-3 / 4}
$$

where $c_{\text {ion }}\left(=c_{\mathrm{s}}+c_{\text {res }}\right)$ is the total molar concentration of salts $\left(c_{\text {ion }}=c_{\text {res }}\right.$ in salt-free case $) . A$ is the number of monomers between uncondensed charges and estimated as $A=2$, determined through osmotic pressure measurements in the SUF regime (see details in Section 2.5). The specific viscosity data was fitted with eq 5 (the blue curve in Figure $3 b$ ). We obtained $c_{\text {res }}=1.1 \mathrm{mM}$ as the best fit parameter, which also shows good agreement between the specific viscosity data and the scaling prediction in the DF regime (the red curve in Figure $3 b$ )

$$
\eta_{\mathrm{sp}, \mathrm{DF}} \propto c_{\mathrm{p}}\left(r_{\mathrm{B}}\right)^{6 / 5} \propto c_{\mathrm{p}}^{2 / 5}\left[1+\frac{2 A c_{\text {ion }}}{c_{\mathrm{p}}}\right]^{-3 / 5}
$$

where $r_{\mathrm{B}}\left(\propto c_{\mathrm{p}}^{-1 / 2}\left[1+\frac{2 A_{c_{\text {on }}}}{c_{\mathrm{p}}}\right]^{-1 / 2}\right)$ is the electrostatic screening length for polyelectrolyte chains. The good agreement between the measured $\eta_{\mathrm{sp}}$ and the scaling predictions indicates that DMF behaves as a good solvent for $\mathrm{PC}_{4}$-TFSI. Hereafter, the value $A=2$ estimated from osmotic pressure measurements and the value $c_{\text {res }}=1.1 \mathrm{mM}$ estimated through eq 5 will be used to fit the experimental data of the specific viscosity 
(Section 3.2.3) and the longest relaxation time (Section 3.2.4) of $\mathrm{PC}_{4}$-TFSI in the mixture of DMF and Bmim-TFSI, i.e., in the presence of salt.

As a final remark, our polymerized ionic liquid solutions showed no maximum in the reduced viscosity $\eta_{\mathrm{sp}} / c_{\mathrm{p}}$ (blue squares in Figure $3 \mathrm{~b}$ ), whereas a maximum is usually observed near the polymer regime transition from SUF to DF regimes for aqueous polyelectrolyte solutions. ${ }^{25,70-73}$ Similar behavior was reported for vinylpyridinium-based polyelectrolytes dissolved in ethylene glycol. ${ }^{34}$ Although the underlying molecular mechanism is not well understood yet, our experimental data showed good agreement with the scaling prediction in the DF regime

$$
\frac{\eta_{\mathrm{sp}, \mathrm{DF}}}{c_{\mathrm{p}}} \propto c_{\mathrm{p}}^{-3 / 5}\left[1+\frac{2 A c_{\text {ion }}}{c_{\mathrm{p}}}\right]^{-3 / 5}
$$

The measured reduced viscosity was fitted well by eq 7 with $A$ $=2$ and $c_{\text {res }}=1.1 \mathrm{mM}$ (the green curve in Figure $3 \mathrm{~b}$ ).

In summary, our results show that $\mathrm{PC}_{4}$-TFSI in DMF behaves as an ordinary polyelectrolyte in a good solvent, in 'salt free' environment. The shear viscosity is a constant at low shear rates but displays shear-thinning features beyond a critical shear rate, which depends on the polymer concentration. From the specific viscosity, we obtained the overlap concentration $c^{*} \sim 10^{-3} \mathrm{M}$ and the residual salt concentration $c_{\text {res }}=1.1 \mathrm{mM}$.

3.2. Salt-Rich Solutions: $\mathrm{PC}_{4}$-TFSI in a Mixture of DMF and Bmim-TFSI. 3.2.1. Effect of the Solvent Mixture: Solvent Viscosity. In the scaling theory of Dobrynin et al., ${ }^{19}$ the viscoelastic properties are controlled by the electrostatic interactions. This scaling relation has been validated for ordinary polyelectrolyte solutions with low salt concentrations, where the Debye-Hückel theory holds. ${ }^{25,26,28,34-36,66,67}$ However, for the binary solvent of DMF and Bmim-TFSI, the solvent viscosity and the dielectric constant may vary with $c_{s}$, i.e., the concentration of the ionic liquid Bmim-TFSI. Figure 4 shows the dependence of the solvent zero-shear viscosity $\eta_{s}$

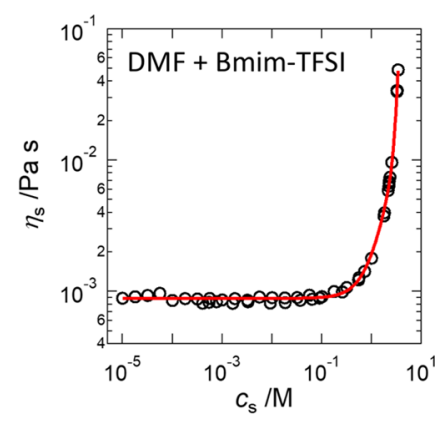

Figure 4. Rapid increase of the solvent viscosity is observed at $c_{\mathrm{s}}>0.1$ M. The solvent viscosity $\eta_{\mathrm{s}}$ is plotted as a function of the concentration of Bmim-TFSI, $c_{s}$. The measured $\eta_{s}$ (black circles) is fitted with the Jones-Dole-type equation (eq 8 , the red solid curve). All measurements are performed at $25^{\circ} \mathrm{C}$.

on $c_{\mathrm{s}}$. The solvent viscosity monotonically increased with increasing $c_{s}$. In particular, there is a sharp increase of $\eta_{s}$ at $c_{\mathrm{s}}>0.1 \mathrm{M}$. Thus, we expect that the viscoelastic properties of $\mathrm{PC}_{4}$-TFSI in the mixture of DMF and Bmim-TFSI would be significantly affected by the change of the solvent viscosity at $c_{\mathrm{s}}>0.1 \mathrm{M}$. Similar trends of $\eta_{\mathrm{s}}$ versus $c_{\mathrm{s}}$ were observed for solutions containing ionic liquids ${ }^{74-78}$ or inorganic salts, such as sodium chloride. ${ }^{79-82}$ The viscosity increase of salt solutions results from the strong interactions among ions, ${ }^{83}$ implying the breakdown of the scaling theory proposed by Dobrynin et al. at $c_{\mathrm{s}}>0.1 \mathrm{M}$ (see details in Section 3.2.2). ${ }^{19}$

Among theoretical and empirical models proposed in literature, ${ }^{74-76,78,81,83}$ we employed a modified Jones-Doletype equation $^{83}$ to correlate the measured solvent viscosity with the Bmim-TFSI concentration. The Jones-Dole equation $\left[\eta_{\mathrm{s}}=\eta_{\mathrm{DMF}}\left(1+B c_{\mathrm{s}}^{1 / 2}+D c_{\mathrm{s}}\right)\right]$ is well known as an empirical equation to capture the viscosity of salt solutions at relatively low concentrations. To describe the viscosity for concentrated salt solutions, the Jones-Dole equation has been modified by adding terms with different power exponents of the salt concentration. ${ }^{83}$ For our solvent mixtures, the best fit (the red curve in Figure 4) was given by

$$
\eta_{\mathrm{s}}=\eta_{\mathrm{DMF}}\left(1+B c_{\mathrm{s}}^{1 / 2}+D c_{\mathrm{s}}+E c_{\mathrm{s}}^{2}+F c_{\mathrm{s}}^{10}\right)
$$

where $\eta_{\mathrm{DMF}}=8.8 \times 10^{-4} \mathrm{~Pa} \cdot \mathrm{s}$ is the viscosity of DMF and $B=0, D=0.11, E=1.1$, and $F=1.8 \times 10^{-4}$ are the best fit coefficients. Shear viscosity curves for the mixture of DMF and Bmim-TFSI are provided in Figure S6 of the Supporting Information. Bear in mind that the solvent viscosity is the viscosity of the mixture of DMF and Bmim-TFSI in this section.

The relative dielectric constant $\epsilon_{\mathrm{r}}$ of the solvent mixture can vary with $c_{s}$. Stoppa et al. ${ }^{84}$ reported a maximum in the dielectric constant $\left(\epsilon_{\mathrm{r}, \max }=38\right)$ at intermediate ionic liquid concentration for a mixture of an ionic liquid, 1-butyl-3methylimidazolium tetrafluoroborate $\left(\epsilon_{\mathrm{r}}=15\right)$, and acetonitrile $\left(\epsilon_{\mathrm{r}}=36\right)$. Since the value of $\epsilon_{\mathrm{r}}$ for each component is similar to that for the mixture of DMF $\left(\epsilon_{\mathrm{r}}=37\right)$ and BmimTFSI $\left(\epsilon_{\mathrm{r}}=16\right)$, we assume that the trend of $\epsilon_{\mathrm{r}}$ versus $c_{\mathrm{s}}$ for our solvent mixture is similar to that observed by Stoppa et al., ${ }^{84}$ indicating that the value of $\epsilon_{\mathrm{r}}$ of the solvent mixture can vary at most by a factor of 2 . In contrast, the solvent viscosity varies almost 2 orders of magnitude with increasing salt concentrations (Figure 4). Moreover, the scaling laws for the specific viscosity and the longest relaxation time predict that the power index for dielectric constant is smaller than the power indexes for the other varying factors. ${ }^{19}$ For these reasons, we neglect the variation of $\epsilon_{\mathrm{r}}$ with $c_{\mathrm{s}}$ in our analysis.

3.2.2. Scaling Laws for Polyelectrolytes in Concentrated Salt Solutions. The scaling laws of $\eta_{\text {sp }}$ (eqs 5 and 6) and the longest relaxation time $\lambda\left(\propto \eta_{\mathrm{s}} \eta_{\mathrm{sp}} c_{\mathrm{p}}^{-1}\right)$ for ordinary polyelectrolyte solutions reported in Dobrynin et al. ${ }^{19}$ are based on the Debye-Hückel theory, ${ }^{33}$ which is valid only at relatively low salt concentrations where ion-ion interactions are negligible. Therefore, the screening length used in the scaling laws is likely to be invalid for $\mathrm{PC}_{4}$-TFSI solutions at $c_{\mathrm{s}}>0.1 \mathrm{M}$. In fact, several research groups have recently reported the unexpected long screening length in concentrated salt solutions, by employing surface force measurement techniques. ${ }^{37-45}$ Gebbie et $\mathrm{al}^{37}$ first reported that the screening length in pure ionic liquids was much larger than the value predicted by the Debye-Hückel theory. Smith et al. ${ }^{43}$ also observed the long screening length in concentrated solutions of other salt types, such as sodium chloride. They showed the onset of the screening length increase at sufficiently large salt concentrations.

Very recently, Lee et al. $^{44}$ introduced a simple scaling argument for the screening length in concentrated salt solutions, $r_{\mathrm{D}}^{*}$, as a function of the Debye screening length $r_{\mathrm{D}}$ 

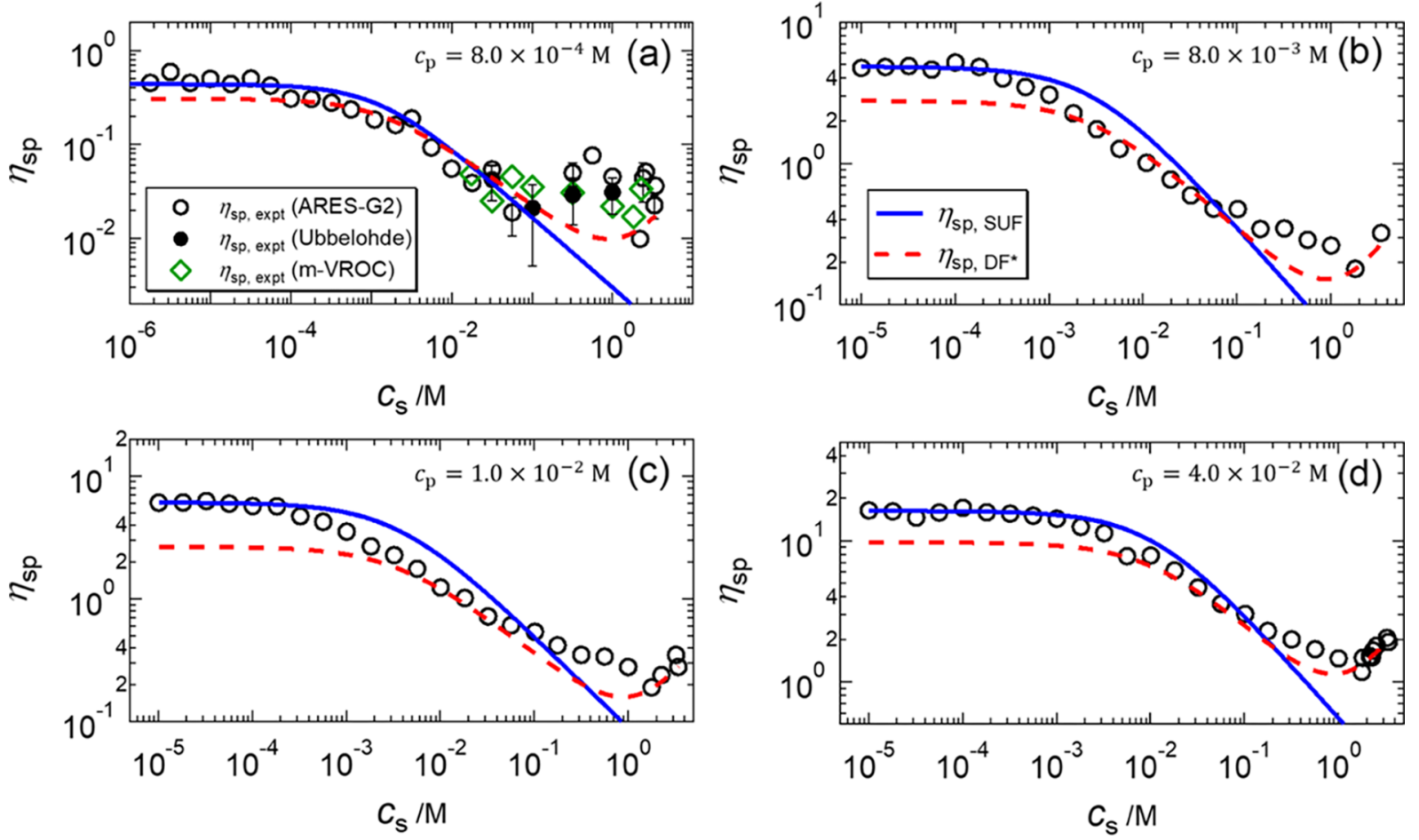

Figure 5. Specific viscosity as a function of the concentration of Bmim-TFSI for PC $\mathrm{PC}_{4}$-TFSI solutions at (a) $c_{\mathrm{p}}=8.0 \times 10^{-4} \mathrm{M},(\mathrm{b}) c_{\mathrm{p}}=8.0 \times 10^{-3}$ $\mathrm{M},(\mathrm{c}) c_{\mathrm{p}}=1.0 \times 10^{-2} \mathrm{M}$, and (d) $c_{\mathrm{p}}=4.0 \times 10^{-2} \mathrm{M}$. The values of $\eta_{\mathrm{sp}}$ via a bulk shear rheometer (ARES-G2; open circles), a microfluidic-based rheometer (m-VROC; open diamonds), and a gravity-driven capillary viscometer (Ubbelohde; filled circles) are compared. Blue solid and red dashed curves represent the scaling prediction of $\eta_{\mathrm{sp}}$ for semidilute unentangled (SUF) and DF* solutions, respectively, with $A=2, c_{\mathrm{res}}=1.1 \mathrm{mM}$ (derived from the fit of the data in "salt-free" environment), and $\epsilon_{\mathrm{r}}=16$. The error bars are omitted when the size of bars is smaller than the size of symbols. The scaling laws are summarized in Table 1 .

Table 1. Scaling Laws of the Specific Viscosity $\eta_{\text {sp }}$ and the Longest Relaxation Time $\lambda$ for Flexible Polyelectrolyte Solutions in the Semidilute Unentangled (SUF) and Dilute (DF) Regimes before/after the Modification with the Adjusted Screening Length Proposed by Lee et al. ${ }^{44}$

\begin{tabular}{|c|c|c|}
\hline polymer regime & specific viscosity $\eta_{\mathrm{sp}}$ & longest relaxation time $\lambda$ \\
\hline SUF & $\eta_{\mathrm{sp}, \mathrm{SUF}} \propto c_{\mathrm{p}}^{1 / 2}\left[1+\frac{2 A c_{\text {ion }}}{c_{\mathrm{p}}}\right]^{-3 / 4}$ & $\lambda_{\mathrm{SUF}} \propto \eta_{\mathrm{s}} c_{\mathrm{p}}^{-1 / 2}\left[1+\frac{2 A c_{\text {ion }}}{c_{\mathrm{p}}}\right]^{-3 / 4}$ \\
\hline $\mathrm{DF}$ & $\eta_{\mathrm{sp}, \mathrm{DF}} \propto c_{\mathrm{p}}^{2 / 5}\left[1+\frac{2 A c_{\mathrm{ion}}}{c_{\mathrm{p}}}\right]^{-3 / 5}$ & $\lambda_{\mathrm{DF}} \propto \eta_{\mathrm{s}} c_{\mathrm{p}}^{-3 / 5}\left[1+\frac{2 A c_{\text {ion }}}{c_{\mathrm{p}}}\right]^{-3 / 5}$ \\
\hline modified DF (DF*) & $\eta_{\mathrm{sp}, \mathrm{DF}} \propto c_{\mathrm{p}}\left[F_{\mathrm{ion}}\left(F_{\mathrm{ion}}^{-3 / 2}+a^{3}\right)\right]^{6 / 5 a}$ & $\lambda_{\mathrm{DF}^{*}} \propto \eta_{\mathrm{s}}\left[F_{\mathrm{ion}}\left(F_{\mathrm{ion}}^{-3 / 2}+a^{3}\right)\right]^{6 / 5}$ \\
\hline$F_{\text {ion }}=\frac{4 \pi N_{\mathrm{A}} l_{\mathrm{B}} c_{\mathrm{p}}}{A}\left(1+2 A c_{\text {ion }} / c_{\mathrm{p}}\right)$. & & \\
\hline
\end{tabular}

$$
\begin{aligned}
& r_{\mathrm{D}}^{*}=r_{\mathrm{D}}\left(1+\frac{a^{3}}{r_{\mathrm{D}}^{3}}\right) \\
& r_{\mathrm{D}}=\left[\frac{4 \pi N_{\mathrm{A}} l_{\mathrm{B}} c_{\mathrm{p}}}{A}\left(1+\frac{2 A c_{\text {ion }}}{c_{\mathrm{p}}}\right)\right]^{-1 / 2} \equiv F_{\text {ion }}^{-1 / 2}
\end{aligned}
$$

where $a$ is the ion diameter and considered to be independent of the salt concentration. $N_{\mathrm{A}}$ and $l_{\mathrm{B}}=\frac{e^{2}}{4 \pi \epsilon_{0} \epsilon_{\mathrm{r}} k_{\mathrm{B}} T}$ are the Avogadro constant and the Bjerrum length, respectively. Here, $e, \epsilon_{0}, k_{\mathrm{B}}$, and $T$ are the elementary charge, the dielectric constant of vacuum, the Boltzmann constant, and the absolute temperature, respectively. We used $F_{\text {ion }}=\frac{4 \pi N_{A} l_{\mathrm{B}} c_{\mathrm{p}}}{A}\left(1+\frac{2 A c_{\text {ion }}}{c_{\mathrm{p}}}\right)$ to simplify the notation.

On the basis of the modified screening length $r_{D}^{*}$ in eq 9, we extend the scaling laws of Dobrynin et al. to concentrated salt solutions. In a salt-concentrated environment, i.e., high $c_{\mathrm{s}}$ polymer solutions are more likely to fall in the DF regime due to the shrinkage of the polymer coil. ${ }^{19}$ Bear this in mind, we replace the expression of the electrostatic screening length $r_{\mathrm{B}}$ in eq 6 with the adjusted screening length $r_{\mathrm{D}}^{*}$ in eq 9 . By doing this, we neglect the electrostatic interactions between polymer chains, appropriate for the case of the DF regime. We then derive the modified scaling laws for the specific viscosity $\eta_{\text {sp, DF* }}$ and the longest relaxation time $\lambda_{\mathrm{DF}^{*}}$ in the modified $\mathrm{DF}$ regime, indicated as $\mathrm{DF}^{*}$

$$
\begin{aligned}
& \eta_{\mathrm{sp}, \mathrm{DF}}{ }^{*} \propto c_{\mathrm{p}}\left(r_{\mathrm{D}}^{*}\right)^{6 / 5} \propto c_{\mathrm{p}}\left[F_{\mathrm{ion}}\left(F_{\mathrm{ion}}^{-3 / 2}+a^{3}\right)\right]^{6 / 5} \\
& \lambda_{\mathrm{DF}^{*}} \propto \eta_{\mathrm{s}}\left(r_{\mathrm{D}}^{*}\right)^{6 / 5} \propto \eta_{\mathrm{s}}\left[F_{\text {ion }}\left(F_{\text {ion }}^{-3 / 2}+a^{3}\right)\right]^{6 / 5}
\end{aligned}
$$

This revised scaling is able to capture our experimental results very well (Figures 5-8); see more details in the subsections below. The good agreement with the modified scaling laws implies that the total molar concentration of salts $c_{\text {ion }}$ decreases 

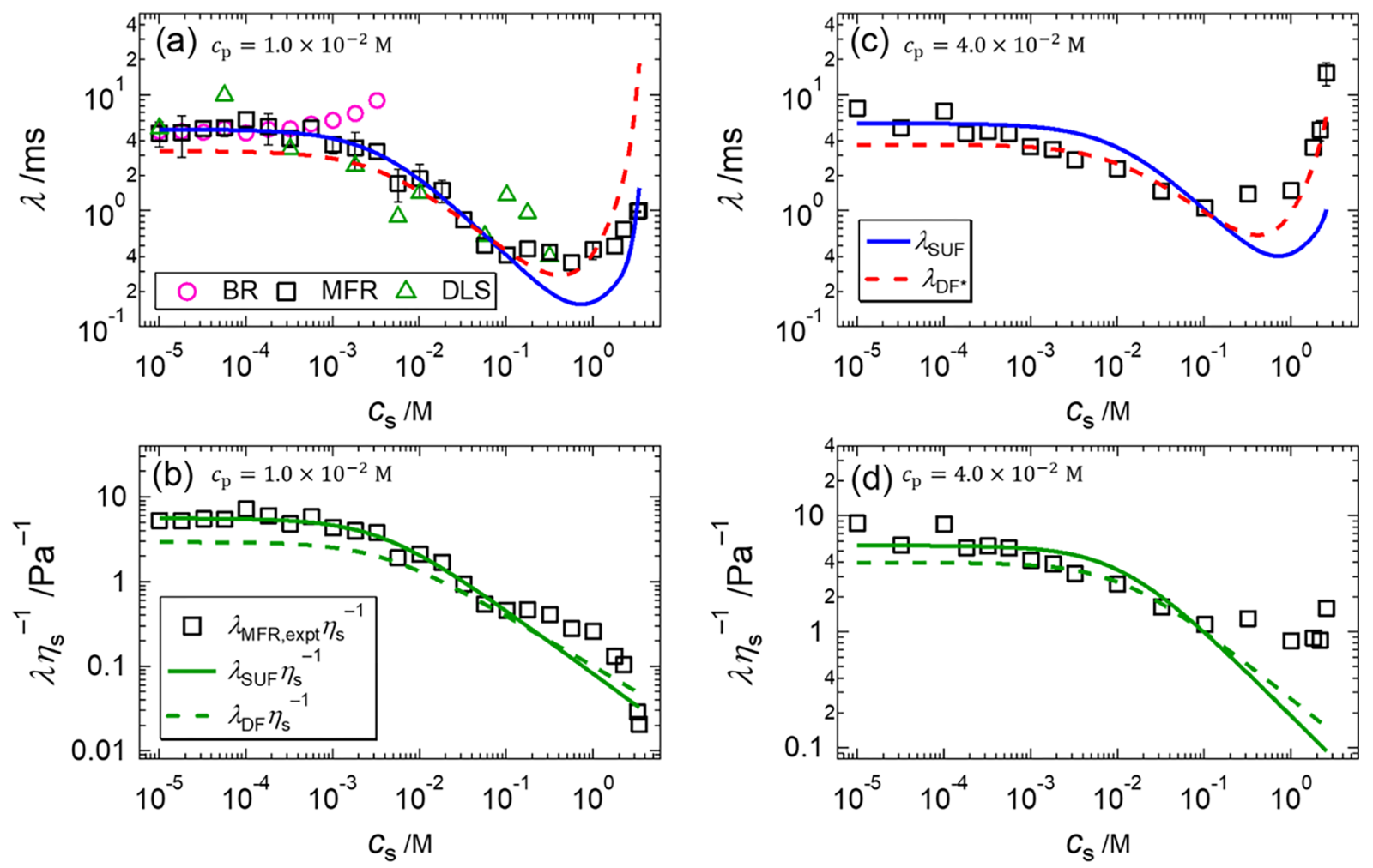

Figure 6. Longest relaxation time $\lambda$ and $\eta_{\mathrm{s}}$-normalized longest relaxation time $\left(\lambda / \eta_{\mathrm{s}}\right)$ as a function of the Bmim-TFSI concentration $c_{\mathrm{s}}$ for PC $\mathrm{C}_{4}$-TFSI solutions at $(\mathrm{a}, \mathrm{b}) c_{\mathrm{p}}=1.0 \times 10^{-2} \mathrm{M}$ and $(\mathrm{c}, \mathrm{d}) c_{\mathrm{p}}=4.0 \times 10^{-2} \mathrm{M}$. The values of $\lambda$ via bulk rheology measurements (BR; magenta circles), dynamic light scattering (DLS; green triangles), and the $\mu$-rheometer (MFR; black squares) are compared. Blue solid and red dashed curves represent the scaling and modified scaling prediction of $\lambda$ for SUF and DF* solutions, respectively. Solid and dashed green curves represent the scaling prediction of $\left(\lambda / \eta_{\mathrm{s}}\right)$ for SUF and DF solutions, respectively. In fitting, $A=2, c_{\mathrm{res}}=1.1 \mathrm{mM}, a=4.2 \mathrm{~nm}$, and $\epsilon_{\mathrm{r}}=16$ are used. The error bars are omitted when the size of error bars is smaller than the size of symbols. The scaling laws are summarized in Table 1.

with increasing IL concentrations. The scaling laws for the specific viscosity and the longest relaxation time in the SUF and DF regimes before/after the modification are summarized in Table 1 . Please note that the modified scaling laws in the DF* regime also recover the scaling laws of Dobrynin et al. at low salt concentration limit, i.e., when the Debye-Hückel theory is valid.

3.2.3. Specific Viscosity $\eta_{s p}$. To examine the effect of the charge screening on the viscoelastic properties of $\mathrm{PC}_{4}$-TFSI in the mixture of DMF and Bmim-TFSI, we measured the specific viscosity $\eta_{\text {sp }}$ as a function of the concentration of Bmim-TFSI, $c_{\mathrm{s}}$. Here, Bmim-TFSI acts as both a solvent and a source of salt ions to regulate the charge screening. Therefore, the values of $\eta_{\mathrm{s}}$ shown in Figure 4 were used to calculate the specific viscosity at each Bmim-TFSI concentration. We tested four $\mathrm{PC}_{4}$-TFSI solutions at four different monomer concentrations: $c_{\mathrm{p}}=8.0 \times 10^{-4}, 8.0 \times 10^{-3}, 1.0 \times 10^{-2}$, and $4.0 \times 10^{-2} \mathrm{M}$.

At the smallest polymer concentration $c_{\mathrm{p}}=8.0 \times 10^{-4} \mathrm{M}$ (Figure 5a), the values of $\eta_{\text {sp }}$ were always below (but close to) the unity, suggesting that the chains were relatively isolated in the mixture over the measured $c_{\mathrm{s}}$ range (i.e., in the DF polymer regime). The specific viscosity was initially a constant until $c_{s}$ reached the value $c_{\mathrm{s}}^{*}=5.0 \times 10^{-5} \mathrm{M}$, beyond which the value of $\eta_{\text {sp }}$ decreased with increasing $c_{\mathrm{s}}$. Similar behavior has been reported for ordinary polyelectrolytes in a good solvent, $^{25,35,36,67}$ suggesting that ionic liquid ions screen out the charges on the $\mathrm{PC}_{4}$-TFSI chain by the similar mechanism to that in ordinary salts, such as sodium chloride. The critical salt concentration $c_{\mathrm{s}}^{*}$ marks the transition between the low-salt and high-salt limits in the DF regime and can be estimated as the Bmim-TFSI concentration at which the number density of uncondensed charges is equal to the number density of BmimTFSI ions, i.e., $c_{\mathrm{s}}^{*}=c_{\mathrm{p}} / 2 A$. This estimate yields $8.0 \times 10^{-5} \mathrm{M}$, in good agreement with the experimentally observed value of $5.0 \times 10^{-5} \mathrm{M}$. At $c_{\mathrm{s}}>1.0 \mathrm{M}$, the value of $\eta_{\mathrm{sp}}$ increased with increasing $c_{\mathrm{s}}$.

By increasing the polymer concentration to $c_{\mathrm{p}}=8.0 \times$ $10^{-3} \mathrm{M}$ (Figure $5 \mathrm{~b}$ ), the value of $\eta_{\mathrm{sp}}$ in the low-salt regime remained a constant and above unity (i.e., in the SUF polymer regime). By increasing $c_{s}$, the specific viscosity decreased below unity across $c_{\mathrm{s}}^{*} \sim 1.0 \times 10^{-2} \mathrm{M}$, thus marking a transition from the SUF to the DF polymer regimes. However, beyond $c_{\mathrm{s}} \sim$ $1.0 \mathrm{M}, \eta_{\mathrm{sp}}$ increased again. This nonmonotonic trend of $\eta_{\mathrm{sp}}$ versus $c_{\mathrm{s}}$ was also observed at higher polymer concentrations of $c_{\mathrm{p}}=1.0 \times 10^{-2} \mathrm{M}$ (Figure 5c) and $c_{\mathrm{p}}=4.0 \times 10^{-2} \mathrm{M}$ (Figure 5d): (i) $\eta_{\text {sp }}$ remains constant at low $c_{s}$, (ii) $\eta_{\text {sp }}$ decreases with increasing $c_{\mathrm{s}}$ at an intermediate $c_{s}$, in agreement with the behavior of ordinary polyelectrolytes in a good solvent, and (iii) $\eta_{\mathrm{sp}}$ increases at $c_{\mathrm{s}}>1.0 \mathrm{M}$.

We compared the measured $\eta_{\text {sp }}$ dependence on $c_{s}$ with the scaling predictions for polyelectrolyte solutions in the SUF polymer regime ${ }^{19}$ (blue solid curves) and with the modified scaling predictions in the $\mathrm{DF}^{*}$ polymer regime (red dashed curves) in Figure 5. Here, the total salt concentration $c_{\text {ion }}$ is the sum of molar concentrations of Bmim-TFSI and residual salts, i.e., $c_{\text {ion }}=c_{s}+c_{\text {res. }}$. The parameter $A=2$ related to the effective charge on $\mathrm{PC}_{4}$-TFSI chains and the residual salt concentration $c_{\text {res }}=1.1 \mathrm{mM}$ are independent of both $c_{\mathrm{s}}$ and $c_{\mathrm{p}}$, as the polymer 

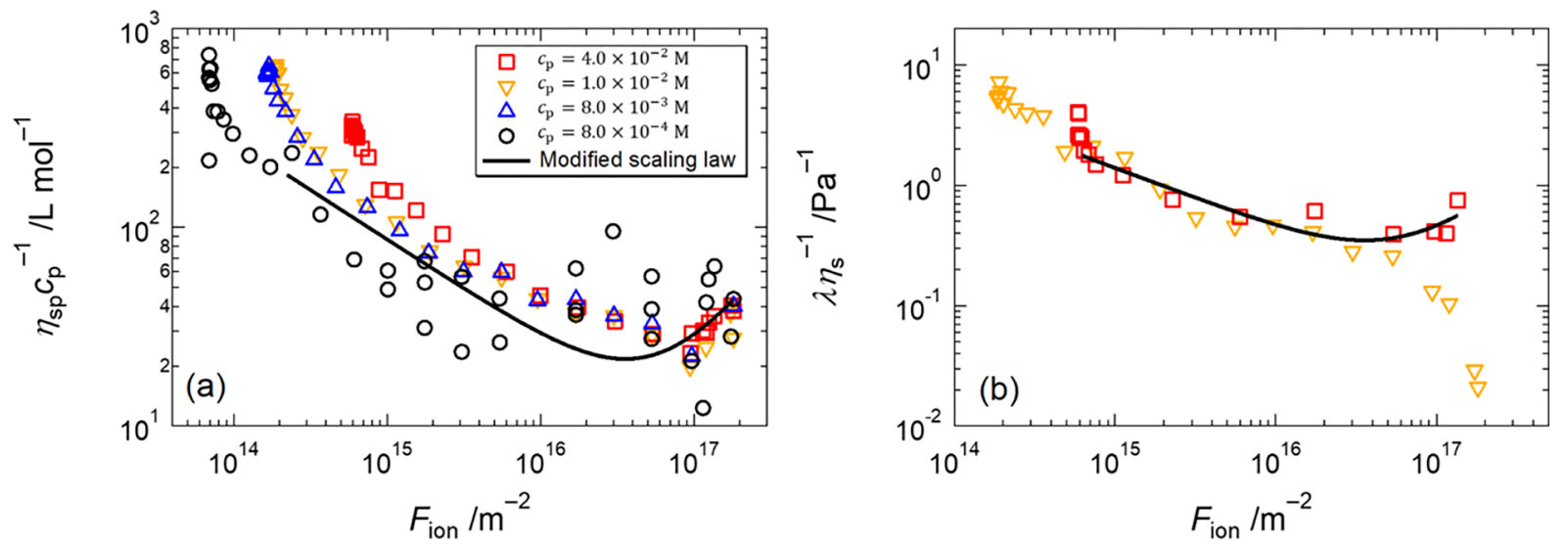

Figure 7. (a) Reduced viscosity $\eta_{\mathrm{sp}} / c_{\mathrm{p}}$ is plotted against the inverse square of the Debye length $F_{\text {ion }}$ at $c_{\mathrm{p}}=4.0 \times 10^{-2} \mathrm{M}(\mathrm{squares}), c_{\mathrm{p}}=1.0 \times 10^{-2}$ $\mathrm{M}$ (inverted triangles), $c_{\mathrm{p}}=8.0 \times 10^{-3} \mathrm{M}$ (triangles), and $c_{\mathrm{p}}=8.0 \times 10^{-4} \mathrm{M}$ (inverted triangles). The solid curve represents the modified scaling prediction for DF* solutions (eq 11) with $a=4.2 \mathrm{~nm}$. (b) Longest relaxation time normalized by the solvent viscosity $\lambda / \eta_{\mathrm{s}}$ is plotted against $F_{\text {ion }}$ at $c_{\mathrm{p}}=4.0 \times 10^{-2} \mathrm{M}$ (squares) and $c_{\mathrm{p}}=1.0 \times 10^{-2} \mathrm{M}$ (inverted triangles). The solid curve represents the modified scaling prediction for $\mathrm{DF}^{*}$ solutions (eq 12) with $a=4.2 \mathrm{~nm}$.

concentration regime is in close proximity to SUF. Indeed, only at very high polymer concentrations, Bordi et al. ${ }^{50}$ observed a change of $A$ with the polymer concentration. The value of $\epsilon_{\mathrm{r}}$ was chosen as $\epsilon_{\mathrm{r}}=16$ for Bmim-TFSI since the modified scaling predictions in the $\mathrm{DF}^{*}$ regime were applied to fit the experimental data at higher $c_{s}$. Therefore, the only adjustable parameter in the curve fitting is the proportionality coefficient, which gives the vertical shift of the predicted curve and the ion diameter $a$. At $c_{\mathrm{p}}=8.0 \times 10^{-4} \mathrm{M}$ (Figure 5a), the data in the low-salt limit were better described by the scaling prediction in the SUF regime, rather than by that for the DF regime. Therefore, polymer chains still interacted with each other despite $\eta_{\mathrm{sp}}<1$. This further implies that the transition between different polymer regimes is never sharp. Beyond the salt concentration $c_{\mathrm{s}}^{*} \sim 1.0 \times 10^{-4} \mathrm{M}$, experimental data were well described only by the modified scaling in the $\mathrm{DF}^{*}$ regime. Similar trends were also observed for all of the other polymer concentrations (Figure $5 \mathrm{~b}-\mathrm{d}$ ).

The value of $a$ was obtained as $a=4.2 \mathrm{~nm}$. In the scaling analysis for the screening length to derive eq 9, Lee et al. ${ }^{44}$ estimated the ion diameter of ionic liquid ions in solutions the same as that in pure ionic liquids, i.e., $c_{\text {pair }}^{-1 / 3} / 2$. Here, $c_{\text {pair }}$ is the ion pair concentration, calculated by $c_{\text {pair }}=N_{\mathrm{A}} c_{\mathrm{s}}$. This argument gives $a=3.9 \mathrm{~nm}$ for Bmim-TFSI, close to $a=4.2 \mathrm{~nm}$ obtained in our study.

We demonstrated that our experimental data agree remarkably well with the scaling prediction by Dobrynin et al. in the SUF regime for solutions at lower $c_{\mathrm{s}}$ and the modified scaling prediction in the $\mathrm{DF}^{*}$ regime for solutions at higher $c_{s}$. We remind the reader that our modified scaling laws also account for the DF regime at low salt concentrations in which the Debye-Hückel theory applies (Section 3.2.2).

3.2.4. Longest Relaxation Time $\lambda$. The longest relaxation time $\lambda$ provides important information on the polymer contribution to the viscoelasticity of the solution. The value of $\lambda$ is solvent dependent, generally higher in more viscous solvents. ${ }^{56}$ Based on the result of the previous sections, we expect $\lambda$ to first decrease with increasing $c_{\mathrm{s}}$ up to $c_{\mathrm{s}} \sim 0.1 \mathrm{M}$ (as predicted by Dobrynin et al. ${ }^{19}$ ) and then increase with increasing $c_{\mathrm{s}}$ due to the ion-ion interaction in the solvent.

We measured $\lambda$ of $\mathrm{PC}_{4}$-TFSI in the mixture of DMF and Bmim-TFSI, at two fixed polymer concentrations of $c_{\mathrm{p}}=1.0 \times$
$10^{-2}$ and $4.0 \times 10^{-2} \mathrm{M}$. We employed three complementary measurement techniques: bulk rheology (BR), dynamic light scattering (DLS) microrheology, and the $\mu$-rheometer (microfilament rheometer, MFR) techniques for comparison purposes. At $c_{\mathrm{p}}=1.0 \times 10^{-2} \mathrm{M}$ (Figure 6a), the agreement among the $\lambda$ values measured through these three methods was striking when $c_{\mathrm{s}}<10^{-3} \mathrm{M}$. However, above $c_{\mathrm{s}}>10^{-3} \mathrm{M}$, the BR data departed from those of MFR and microrheology due to the sensitivity limit of the rheometer (see more discussion on the rheometer sensitivity in Section S5.2 of the Supporting Information). The longest relaxation time $\lambda$ was initially a constant up to $c_{\mathrm{s}}=3.0 \times 10^{-4} \mathrm{M}$ and then decreased with increasing $c_{\mathrm{s}}$ until $c_{\mathrm{s}} \sim 0.1 \mathrm{M}$. At $c_{\mathrm{s}}>1 \mathrm{M}, \lambda$ increased again, displaying the same nonmonotonic behavior as that of the specific viscosity. Similar behavior was also observed for solutions at $c_{\mathrm{p}}=4.0 \times 10^{-2} \mathrm{M}$ (Figure $6 \mathrm{c}$ ). Moreover, the terminal modulus, calculated by $\eta_{0} / \lambda$, was independent of $c_{\mathrm{s}}$ for both $c_{\mathrm{p}}=1.0 \times 10^{-2}$ and $4.0 \times 10^{-2} \mathrm{M}$ (see Figure S7 of the Supporting Information).

Recall that $\lambda$ is predicted to increase with increasing $\eta_{s}$ (solvent viscosity) based on the scaling laws (see Table 1 ). To evaluate the effect of elevated $\eta_{\mathrm{s}}$ at high $c_{\mathrm{s}}$ (see Figure 4) on $\lambda$, we plotted the longest relaxation time normalized by the solvent viscosity, $\left(\lambda / \eta_{\mathrm{s}}\right)$, as a function of $c_{\mathrm{s}}$. The values of $\lambda / \eta_{\mathrm{s}}$ at $c_{\mathrm{p}}=1.0 \times 10^{-2} \mathrm{M}$ (Figure $6 \mathrm{~b}$ ) and $c_{\mathrm{p}}=4.0 \times 10^{-2} \mathrm{M}$ (Figure 6d) were initially a constant up to $c_{\mathrm{s}} \sim 1.0 \times 10^{-3} \mathrm{M}$ and then decreased with increasing $c_{\mathrm{s}}$ until $c_{\mathrm{s}} \sim 1.0 \times 10^{-1} \mathrm{M}$, in good agreement with the scaling predictions, as indicated by two fitting curves for SUF (green solid curve) and DF (green dashed curve) solutions with $A=2$ and $c_{\text {res }}=1.1 \mathrm{mM}$. However, the experimental data of $\lambda / \eta_{\mathrm{s}}$ deviated from the scaling predictions at $c_{\mathrm{s}}>1.0 \times 10^{-1} \mathrm{M}$.

This discrepancy at $c_{\mathrm{s}}>1.0 \times 10^{-1} \mathrm{M}$ can be captured by the modified scaling law for DF* solutions (eq 12). In Figure 6a,c, we compared the measured $\lambda$ dependence on $c_{s}$ with the scaling prediction for SUF solutions (blue solid curves) and the modified scaling prediction for $\mathrm{DF}^{*}$ solutions (red dashed curves). The value of $a$ was fixed as $a=4.2 \mathrm{~nm}$ from the fitting of $\eta_{\mathrm{sp}}$; therefore, the only adjustable parameter was the proportionality coefficient, which determines the vertical position of the curve. Our experimental data showed good 
agreement with the predictions over the whole range of salt concentrations $c_{\mathrm{s}}$ examined.

We further test the validity of the modified scaling laws based on eqs 11 and 12. Figure 7a shows the dependence of the measured reduced viscosity $\eta_{\mathrm{sp}} / c_{\mathrm{p}}$ on the inverse square of the Debye length $F_{\text {ion }}$ at different monomer concentrations $c_{\mathrm{p}}$. If the modified scaling law were valid, $\eta_{\mathrm{sp}} / c_{\mathrm{p}}$ versus $F_{\text {ion }}$ should be reduced to a single curve since the polymer solutions are in the DF* regime at high $c_{s}$. As shown in Figure $7 \mathrm{a}$, regardless of $c_{\mathrm{p}}$, the value of $\eta_{\mathrm{sp}} / c_{\mathrm{p}}$ decreased with increasing $F_{\text {ion }}$ until $F_{\text {ion }}=10^{17} \mathrm{~m}^{-2}$, across which the value of $\eta_{\mathrm{sp}} / c_{\mathrm{p}}$ increased with increasing $F_{\text {ion. }}$. At low $F_{\text {ion }}$ the data at different $c_{\mathrm{p}}$ disagreed with each other because the solutions belong to the SUF regime. On the other hand, the data at higher $F_{\text {ion }}$ ranging from $3.0 \times 10^{15}$ to $2.0 \times 10^{17} \mathrm{~m}^{-2}$ collapsed into a single curve, validating the modified scaling law proposed in this study. Moreover, the trend of $\eta_{\mathrm{sp}} / c_{\mathrm{p}}$ versus $F_{\text {ion }}$ agreed well with the scaling prediction (eq 11) with $a=4.2 \mathrm{~nm}$. The reduced plot was also obtained for the dependence of the solvent viscosity normalized longest relaxation time $\lambda / \eta_{\mathrm{s}}$ on $F_{\text {ion }}$ (Figure $7 \mathrm{~b}$ ). We observed some discrepancy at $F_{\text {ion }}>10^{17} \mathrm{~m}^{-2}$ for the solution at $c_{\mathrm{p}}=1.0 \times 10^{-2} \mathrm{M}$, possibly caused by the polymer regime transition. Nevertheless, good agreement between the measured $\lambda / \eta_{\mathrm{s}}$ at $c_{\mathrm{p}}=4.0 \times 10^{-2} \mathrm{M}$ and the scaling prediction (eq 12) validated the modified scaling laws proposed in this study. Note that the data at $c_{\mathrm{p}}=4.0 \times 10^{-2}$ and $4.0 \times 10^{-2} \mathrm{M}$ were vertically shifted slightly in accord with the proportionality coefficient in eqs 11 and 12 .

3.3. Hydrodynamic Radius $\boldsymbol{R}_{\mathrm{H}}$. The good agreement of the modified scaling laws with the experimental data is consistent with our assumption that the screening length increases with increasing Bmim-TFSI concentration, resulting in the chain expansion at $c_{\mathrm{s}}>1.0 \mathrm{M}$. We therefore measured the hydrodynamic radius $R_{\mathrm{H}}$ for $\mathrm{PC}_{4}$-TFSI solutions at $c_{\mathrm{p}}=1.0 \times 10^{-2} \mathrm{M}$ by dynamic light scattering. The BmimTFSI concentration range was chosen as $0.1 \mathrm{M}<c_{s} \leq 3.42 \mathrm{M}$, where the solutions fall under the DF regime. Figure 8 shows the dependence of $R_{\mathrm{H}}$ on $c_{\mathrm{s}}$, observed at $40^{\circ}$ of scattering angle with an averaging time of $600 \mathrm{~s}$. All measurements were performed at $25{ }^{\circ} \mathrm{C}$. The hydrodynamic radius $R_{\mathrm{H}}$ remained constant at $c_{\mathrm{s}}<1 \mathrm{M}$ but displayed a steady increase beyond $c_{\mathrm{s}} \sim 1 \mathrm{M}$, confirming the increase of the size of the single polymer chain with increasing $c_{s}$.

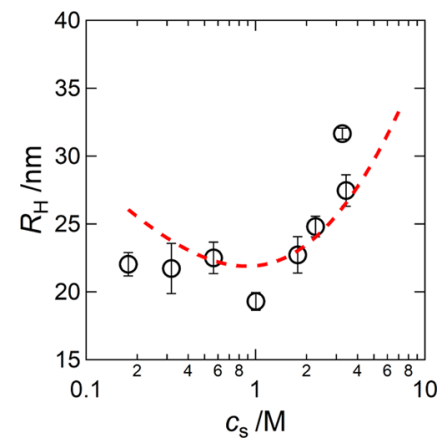

Figure 8. Increase of the hydrodynamic radius $R_{\mathrm{H}}$ for $\mathrm{PC}_{4}$-TFSI solutions at $c_{\mathrm{p}}=1.0 \times 10^{-2} \mathrm{M}$ is observed in the range of $1.0 \mathrm{M}<c_{\mathrm{s}} \leq 3.42 \mathrm{M}$ (DF regime). The value of $R_{\mathrm{H}}$ at $25{ }^{\circ} \mathrm{C}$ was measured by dynamic light scattering (3D LS spectrometer, LS Instruments). The red dashed curve represents the scaling prediction for $\mathrm{DF}^{*}$ solutions (eq 13). The experimental errors are obtained from three independent measurements.
We compared the measured $R_{\mathrm{H}}$ dependence on $c_{\mathrm{s}}$ with the modified scaling for $\mathrm{DF}^{*}$ solutions. The modified scaling relation for $R_{\mathrm{H}}$ can be given by

$$
R_{\mathrm{H}}^{*} \propto R \propto\left(r_{\mathrm{D}}^{*}\right)^{2 / 5} \propto\left[F_{\text {ion }}\left(F_{\text {ion }}^{-3 / 2}+a^{3}\right)\right]^{2 / 5}
$$

where $R$ is the end-to-end distance of polyelectrolytes in a good solvent. The predicted curve with $a=4.2 \mathrm{~nm}$ was in good agreement with the experimental data, thus confirming our assumption that the screening length increases with increasing Bmim-TFSI concentration $c_{\mathrm{s}}$ (also termed as charge underscreening) and further validating the applicability of the modified scaling law in the $\mathrm{DF}^{*}$ regime.

\section{CONCLUSIONS}

In this work, we investigated the effect of the charge screening on the viscoelastic properties and the conformation of polymerized ionic liquids (PILs) in ionic liquid (IL) solutions by probing the specific viscosity $\eta_{\mathrm{sp}}$ and the longest relaxation time $\lambda$ of the polymerized ionic liquid $\mathrm{PC}_{4}$-TFSI in a mixture of DMF and ionic liquid Bmim-TFSI. From the trend of the specific viscosity $\eta_{\mathrm{sp}}$ versus the polymer concentration $c_{\mathrm{p}}$ in salt-free solutions, we estimated the residual salt concentration $c_{\text {res }}=1.1 \mathrm{mM}$ with the number of monomers between uncondensed charges $A=2$ evaluated by osmotic pressure measurements. We then studied the four different polymer concentrations ranging from $c_{\mathrm{p}}=8.0 \times 10^{-4}$ to $4.0 \times 10^{-2} \mathrm{M}$, with the ionic liquid concentration $0 \mathrm{M} \leq c_{\mathrm{s}} \leq 3.42 \mathrm{M}$. Specifically we identified three regions: (i) at low $c_{\mathrm{s}}, \eta_{\mathrm{sp}}$ and $\lambda$ remained constant with increasing $c_{s}$; (ii) at an intermediate $c_{s}$, $\eta_{\text {sp }}$ and $\lambda$ decreased with increasing $c_{\mathrm{s}}$; (iii) at sufficiently high $c_{s}, \eta_{\text {sp }}$ and $\lambda$ increased with increasing $c_{s}$. Regions (i) and (ii) were well described by the scaling laws of Dobrynin et al. for ordinary polyelectrolytes in a good solvent. ${ }^{19}$ We introduced a modified scaling law for $\eta_{\text {sp }}$ and $\lambda$, based on a modified Debye screening length in concentrated salt solutions. ${ }^{44}$ This modified scaling law captured the measured $\eta_{\mathrm{sp}}$ and $\lambda$ in the region (iii) remarkably well. These results indicate that polymerized ionic liquid chains shrink as the ionic liquid concentration increases at lower concentrations due to the charge screening effect by the same mechanism as that for ordinary polyelectrolytes in salt solutions but start to expand at higher ionic liquid concentrations due to the charge underscreening effect. Our results shed new insights on the conformation of PIL in IL solutions and provide, for the first time, a valid reference for the study of general polyelectrolyte solutions at high ion concentrations, where the Debye-Hückel theory is no longer valid.

\section{ASSOCIATED CONTENT}

\section{S Supporting Information}

The Supporting Information is available free of charge on the ACS Publications website at DOI: 10.1021/acs.macromol.8b02544.

Sections S1-S3: details for synthesis and characterization; Section S4: measurement principles for $\mathrm{m}$ VROC, gravity-driven capillary viscometer, and microrheology; Section S5: technical limitation of bulk rheometry; Section S6: density of the mixture of DMF and Bmim-TFSI, shear viscosity curves for mixtures of DMF and Bmim-TFSI, terminal modulus; MATLAB codes are available at http://groups.oist.jp/mbnu (PDF) 


\section{AUTHOR INFORMATION}

\section{Corresponding Authors}

*E-mail: atsushi.matsumoto@oist.jp (A.M.).

*E-mail: amy.shen@oist.jp (A.Q.S.).

\section{ORCID $\odot$}

Atsushi Matsumoto: 0000-0002-8157-3083

Francesco Del Giudice: 0000-0002-9414-6937

Amy Q. Shen: 0000-0002-1222-6264

Notes

The authors declare no competing financial interest.

\section{ACKNOWLEDGMENTS}

The authors thank the anonymous reviewers for their careful review of our manuscript and their insightful comments and suggestions. The authors thank Prof. John de Bruyn from the Department of Physics and Astronomy at the University of Western Ontario, Canada, for helpful suggestions and careful proofreading. The authors also thank Kang-Yu Chu and TingChun Chou from Micro/Bio/Nanofluidics Unit at OIST for their help with developing a custom-made video tracking MATLAB code for our gravity-driven capillary viscometer analysis. The authors gratefully acknowledge the support of the Okinawa Institute of Science and Technology Graduate University with subsidy funding from the Cabinet Office, Government of Japan. A.Q.S. also acknowledges funding from the Japanese Society for the Promotion of Science [Grants-inAid for Scientific Research (C) Grant JP17K06173 and Grants-in-Aid for Scientific Research (B) Grant JP18H01135]. The authors also thank Dr Yoshiteru Iinuma from OIST for his help with the use of the inductively coupled plasma mass spectrometer.

\section{REFERENCES}

(1) Wilkes, J. S.; Zaworotko, M. J. Air and water stable 1-ethyl-3methylimidazolium based ionic liquids. J. Chem. Soc., Chem. Commun. 1992, 965-967.

(2) Yuan, J.; Mecerreyes, D.; Antonietti, M. Poly(ionic liquid)s: An update. Prog. Polym. Sci. 2013, 38, 1009-1036.

(3) Welton, T. Room-Temperature Ionic Liquids. Solvents for Synthesis and Catalysis. Chem. Rev. 1999, 99, 2071-2084.

(4) Wasserscheid, P.; Welton, T. Ionic Liquids in Synthesis, 2nd ed.; John Wiley \& Sons, 2008.

(5) Ohno, H. Electrochemical Aspects of Ionic Liquids; John Wiley \& Sons, 2005.

(6) Cadena, C.; Anthony, J. L.; Shah, J. K.; Morrow, T. I.; Brennecke, J. F.; Maginn, E. J. Why Is $\mathrm{CO}_{2}$ So Soluble in Imidazolium-Based Ionic Liquids? J. Am. Chem. Soc. 2004, 126, 5300-5308.

(7) Zhou, D.; Liu, R.; Zhang, J.; Qi, X.; He, Y.-B.; Li, B.; Yang, Q.H.; Hu, Y.-S.; Kang, F. In situ synthesis of hierarchical poly (ionic liquid)-based solid electrolytes for high-safety lithium-ion and sodium-ion batteries. Nano Energy 2017, 33, 45-54.

(8) Susan, M. A. B. H.; Kaneko, T.; Noda, A.; Watanabe, M. Ion Gels Prepared by in Situ Radical Polymerization of Vinyl Monomers in an Ionic Liquid and Their Characterization as Polymer Electrolytes. J. Am. Chem. Soc. 2005, 127, 4976-4983.

(9) Carlisle, T. K.; McDanel, W. M.; Cowan, M. G.; Noble, R. D.; Gin, D. L. Vinyl-Functionalized Poly(imidazolium)s: A Curable Polymer Platform for Cross-Linked Ionic Liquid Gel Synthesis. Chem. Mater. 2014, 26, 1294-1296.

(10) Swatloski, R. P.; Spear, S. K.; Holbrey, J. D.; Rogers, R. D. Dissolution of Cellose with Ionic Liquids. J. Am. Chem. Soc. 2002, 124, 4974-4975.
(11) Olivier-Bourbigou, H.; Magna, L.; Morvan, D. Ionic liquids and catalysis: Recent progress from knowledge to applications. Appl. Catal., A 2010, 373, 1-56.

(12) Galiński, M.; Lewandowski, A.; Stẹpniak, I. Ionic liquids as electrolytes. Electrochim. Acta 2006, 51, 5567-5580.

(13) Ohno, H.; Ito, K. Room-Temperature Molten Salt Polymers as a Matrix for Fast Ion Conduction. Chem. Lett. 1998, 27, 751-752.

(14) Wood, T. J.; Schofield, W. C.; Lund, P.; Larsen, M. J.; Badyal, J. P. S. Highly ion-conducting poly (ionic liquid) layers. Chem. Commun. 2012, 48, 10201-10203.

(15) Li, M.; Wang, L.; Yang, B.; Du, T.; Zhang, Y. Facile preparation of polymer electrolytes based on the polymerized ionic liquid poly ((4-vinylbenzyl) trimethylammonium bis (trifluoromethanesulfonylimide)) for lithium secondary batteries. Electrochim. Acta 2014, 123, 296-302.

(16) Ni, W.; Liu, S.; Fei, Y.; He, Y.; Ma, X.; Lu, L.; Deng, Y. Preparation of Carbon Nanotubes/Manganese Dioxide Composite Catalyst with Fewer Oxygen-Containing Groups for Li-O2 Batteries Using Polymerized Ionic Liquids as Sacrifice Agent. ACS Appl. Mater. Interfaces 2017, 9, 14749-14757.

(17) Zulfiqar, S.; Sarwar, M. I.; Mecerreyes, D. Polymeric ionic liquids for $\mathrm{CO}_{2}$ capture and separation: potential, progress and challenges. Polym. Chem. 2015, 6, 6435-6451.

(18) Mogurampelly, S.; Ganesan, V. Ion Transport in Polymerized Ionic Liquid-Ionic Liquid Blends. Macromolecules 2018, 51, 94719483.

(19) Dobrynin, A. V.; Colby, R. H.; Rubinstein, M. Scaling Theory of Polyelectrolyte Solutions. Macromolecules 1995, 28, 1859-1871.

(20) Colby, R. H. Structure and linear viscoelasticity of flexible polymer solutions: comparison of polyelectrolyte and neutral polymer solutions. Rheol. Acta 2010, 49, 425-442.

(21) Muthukumar, M. 50th Anniversary Perspective: A Perspective on Polyelectrolyte Solutions. Macromolecules 2017, 50, 9528-9560.

(22) Katchalsky, A. Solutions of polyelectrolytes and mechanochemical systems. J. Polym. Sci. 1951, 7, 393-412.

(23) Khokhlov, A.; Khachaturian, K. On the theory of weakly charged polyelectrolytes. Polymer 1982, 23, 1742-1750.

(24) Araki, T. Conformational changes of polyelectrolyte chains in solvent mixtures. Soft Matter 2016, 12, 6111-6119.

(25) Boris, D. C.; Colby, R. H. Rheology of Sulfonated Polystyrene Solutions. Macromolecules 1998, 31, 5746-5755.

(26) Dou, S.; Colby, R. H. Solution Rheology of a Strongly Charged Polyelectrolyte in Good Solvent. Macromolecules 2008, 41, 65056510.

(27) Nierlich, M.; Williams, C.; Boue, F.; Cotton, J.; Daoud, M.; Famoux, B.; Jannink, G.; Picot, C.; Moan, M.; Wolff, C.; et al. Small angle neutron scattering by semi-dilute solutions of polyelectrolyte. J. Phys. 1979, 40, 701-704.

(28) Lopez, C. G.; Colby, R. H.; Cabral, J. T. Electrostatic and Hydrophobic Interactions in NaCMC Aqueous Solutions: Effect of Degree of Substitution. Macromolecules 2018, 51, 3165-3175.

(29) Pommella, A.; Preziosi, V.; Caserta, S.; Cooper, J. M.; Guido, S.; Tassieri, M. Using Optical Tweezers for the Characterization of Polyelectrolyte Solutions with Very Low Viscoelasticity. Langmuir 2013, 29, 9224-9230.

(30) Oelschlaeger, C.; Cota Pinto Coelho, M.; Willenbacher, N. Chain Flexibility and Dynamics of Polysaccharide Hyaluronan in Entangled Solutions: a High Frequency Rheology and Diffusing Wave Spectroscopy Study. Biomacromolecules 2013, 14, 3689-3696.

(31) Rubinstein, M.; Colby, R. H. Polymer Physics; Oxford University Press: New York, 2003.

(32) de Gennes, P.-G.; Pincus, P.; Velasco, R.; Brochard, F. Remarks on polyelectrolyte conformation. J. Phys. 1976, 37, 1461-1473.

(33) Debye, P.; Hückel, E. The interionic attraction theory of deviations from ideal behavior in solution. Z. Phys. 1923, 24, 185.

(34) Dou, S.; Colby, R. H. Charge density effects in salt-free polyelectrolyte solution rheology. J. Polym. Sci., Part B: Polym. Phys. 2006, 44, 2001-2013. 
(35) Lopez, C. G.; Colby, R. H.; Graham, P.; Cabral, J. T. Viscosity and Scaling of Semiflexible Polyelectrolyte NaCMC in Aqueous Salt Solutions. Macromolecules 2017, 50, 332-338.

(36) Del Giudice, F.; Calcagno, V.; Esposito Taliento, V.; Greco, F.; Netti, P. A.; Maffettone, P. L. Relaxation time of polyelectrolyte solutions: When $\mu$-rheometry steps in charge. J. Rheol. 2017, 61, 1321.

(37) Gebbie, M. A.; Valtiner, M.; Banquy, X.; Fox, E. T.; Henderson, W. A.; Israelachvili, J. N. Ionic liquids behave as dilute electrolyte solutions. Proc. Natl. Acad. Sci. U.S.A. 2013, 110, 9674-9679.

(38) Gebbie, M. A.; Dobbs, H. A.; Valtiner, M.; Israelachvili, J. N. Long-range electrostatic screening in ionic liquids. Proc. Natl. Acad. Sci. U.S.A. 2015, 112, 7432-7437.

(39) Gebbie, M. A.; Smith, A. M.; Dobbs, H. A.; Lee, A. A.; Warr, G. G.; Banquy, X.; Valtiner, M.; Rutland, M. W.; Israelachvili, J. N.; Perkin, S.; Atkin, R. Long range electrostatic forces in ionic liquids. Chem. Commun. 2017, 53, 1214-1224.

(40) Gebbie, M. A.; Valtiner, M.; Banquy, X.; Henderson, W. A.; Israelachvili, J. N. Reply to Perkin et al.: Experimental observations demonstrate that ionic liquids form both bound (Stern) and diffuse electric double layers. Proc. Natl. Acad. Sci. U.S.A. 2013, No. E4122.

(41) Perkin, S.; Salanne, M.; Madden, P.; Lynden-Bell, R. Is a Stern and diffuse layer model appropriate to ionic liquids at surfaces? Proc. Natl. Acad. Sci. U.S.A. 2013, 110, E4121.

(42) Lee, A. A.; Vella, D.; Perkin, S.; Goriely, A. Are RoomTemperature Ionic Liquids Dilute Electrolytes? J. Phys. Chem. Lett. 2015, 6, 159-163.

(43) Smith, A. M.; Lee, A. A.; Perkin, S. The Electrostatic Screening Length in Concentrated Electrolytes Increases with Concentration. J. Phys. Chem. Lett. 2016, 7, 2157-2163.

(44) Lee, A. A.; Perez-Martinez, C. S.; Smith, A. M.; Perkin, S. Scaling Analysis of the Screening Length in Concentrated Electrolytes. Phys. Rev. Lett. 2017, 119, No. 026002.

(45) Lhermerout, R.; Perkin, S. Nanoconfined ionic liquids: Disentangling electrostatic and viscous forces. Phys. Rev. Fluids 2018, 3, No. 014201.

(46) Marcilla, R.; Blazquez, J. A.; Fernandez, R.; Grande, H.; Pomposo, J. A.; Mecerreyes, D. Synthesis of Novel Polycations Using the Chemistry of Ionic Liquids. Macromol. Chem. Phys. 2005, 206, 299-304.

(47) Zimm, B. H. The Scattering of Light and the Radial Distribution Function of High Polymer Solutions. J. Chem. Phys. 1948, 16, 1093-1099.

(48) Wyatt, P. J. Light scattering and the absolute characterization of macromolecules. Anal. Chim. Acta 1993, 272, 1-40.

(49) Manning, G. S. Limiting laws and counterion condensation in polyelectrolyte solutions I. Colligative properties. J. Chem. Phys. 1969, 51, 924-933.

(50) Bordi, F.; Cametti, C.; Tan, J.; Boris, D.; Krause, W.; Plucktaveesak, N.; Colby, R. Determination of Polyelectrolyte Charge and Interaction with Water Using Dielectric Spectroscopy. Macromolecules 2002, 35, 7031-7038.

(51) Konop, A. J.; Colby, R. H. Polyelectrolyte charge effects on solution viscosity of poly (acrylic acid). Macromolecules 1999, 32, 2803-2805.

(52) Payal, R. S.; Bejagam, K. K.; Mondal, A.; Balasubramanian, S. Dissolution of cellulose in room temperature ionic liquids: anion dependence. J. Phys. Chem. B 2015, 119, 1654-1659.

(53) Ewoldt, R. H.; Johnston, M. T.; Caretta, L. M. Complex Fluids in Biological Systems; Springer, 2015.

(54) Rodd, L. E.; Scott, T. P.; Boger, D. V.; Cooper-White, J. J.; McKinley, G. H. The inertio-elastic planar entry flow of low-viscosity elastic fluids in micro-fabricated geometries. J. Non-Newtonian Fluid Mech. 2005, 129, 1-22.

(55) Soulages, J.; Oliveira, M.; Sousa, P.; Alves, M.; McKinley, G. Investigating the stability of viscoelastic stagnation flows in T-shaped microchannels. J. Non-Newtonian Fluid Mech. 2009, 163, 9-24.

(56) Macosko, C. W. Rheology: Principles, Measurements, and Applications; Wiley-VCH: New York, 1994.
(57) Connelly, R. W.; Greener, J. High-Shear Viscometry with a Rotational Parallel-Disk Device. J. Rheol. 1985, 29, 209-226.

(58) Pipe, C. J.; Majmudar, T. S.; McKinley, G. H. High shear rate viscometry. Rheol. Acta 2008, 47, 621-642.

(59) Duda, J. L.; Klaus, E. E.; Lin, S. C. Capillary viscometry study of non-Newtonian fluids: influence of viscous heating. Ind. Eng. Chem. Res. 1988, 27, 352-361.

(60) Gupta, S.; Wang, W. S.; Vanapalli, S. A. Microfluidic viscometers for shear rheology of complex fluids and biofluids. Biomicrofluidics 2016, 10, No. 043402.

(61) Guillot, P.; Panizza, P.; Salmon, J.-B.; Joanicot, M.; Colin, A.; Bruneau, C.-H.; Colin, T. Viscosimeter on a Microfluidic Chip. Langmuir 2006, 22, 6438-6445.

(62) Mason, T. G. Estimating the viscoelastic moduli of complex fluids using the generalized Stokes-Einstein equation. Rheol. Acta 2000, 39, 371-378.

(63) Mason, T. G.; Weitz, D. Optical measurements of frequencydependent linear viscoelastic moduli of complex fluids. Phys. Rev. Lett. 1995, 74, No. 1250.

(64) Haward, S. Microfluidic extensional rheometry using stagnation point flow. Biomicrofluidics 2016, 10, No. 043401.

(65) Del Giudice, F.; D’Avino, G.; Greco, F.; De Santo, I.; Netti, P. A.; Maffettone, P. L. Rheometry-on-a-chip: measuring the relaxation time of a viscoelastic liquid through particle migration in microchannel flows. Lab Chip 2015, 15, 783-792.

(66) Del Giudice, F.; Tassieri, M.; Oelschlaeger, C.; Shen, A. Q. When Microrheology, Bulk Rheology, and Microfluidics Meet: Broadband Rheology of Hydroxyethyl Cellulose Water Solutions. Macromolecules 2017, 50, 2951-2963.

(67) Del Giudice, F.; Haward, S. J.; Shen, A. Q. Relaxation time of dilute polymer solutions: A microfluidic approach. J. Rheol. 2017, 61, $327-337$.

(68) Krause, W. E.; Tan, J. S.; Colby, R. H. Semidilute Solution Rheology of Polyelectrolytes with No Added Salt. J. Polym. Sci., Part B: Polym. Phys. 1999, 37, 3429-3437.

(69) Chen, H.; Elabd, Y. A. Polymerized Ionic Liquids: Solution Properties and Electrospinning. Macromolecules 2009, 42, 3368-3373.

(70) Cohen, J.; Priel, Z.; Rabin, Y. Viscosity of dilute polyelectrolyte solutions. J. Chem. Phys. 1988, 88, 7111-7116.

(71) Eisenberg, H.; Pouyet, J. Viscosities of dilute aqueous solutions of a partially quaternized poly-4-vinylpyridine at low gradients of flow. J. Polym. Sci. 1954, 13, 85-91.

(72) Wyatt, N. B.; Liberatore, M. W. The effect of counterion size and valency on the increase in viscosity in polyelectrolyte solutions. Soft Matter 2010, 6, 3346-3352.

(73) Dubrovskii, S.; Zelenetskii, A.; Uspenskii, S.; Khabarov, V. Effect of borax additives on the rheological properties of sodium hyaluronate aqueous solutions. Polym. Sci., Ser. A 2014, 56, 205-210.

(74) Atashrouz, S.; Zarghampour, M.; Abdolrahimi, S.; Pazuki, G.; Nasernejad, B. Estimation of the Viscosity of Ionic Liquids Containing Binary Mixtures Based on the Eyring's Theory and a Modified Gibbs Energy Model. J. Chem. Eng. Data 2014, 59, 36913704.

(75) Zhao, N.; Jacquemin, J.; Oozeerally, R.; Degirmenci, V. New Method for the Estimation of Viscosity of Pure and Mixtures of Ionic Liquids Based on the UNIFAC-VISCO Model. J. Chem. Eng. Data 2016, 61, 2160-2169.

(76) Cao, Q.; Lu, X.; Wu, X.; Guo, Y.; Xu, L.; Fang, W. Density, Viscosity, and Conductivity of Binary Mixtures of the Ionic Liquid N(2-Hydroxyethyl) Piperazinium Propionate with Water, Methanol, or Ethanol. J. Chem. Eng. Data 2015, 60, 455-463.

(77) Zhao, N.; Jacquemin, J. New method based on the UNIFACVISCO model for the estimation of dynamic viscosity of (ionic liquid + molecular solvent) binary mixtures. Fluid Phase Equilib. 2017, 449, $41-51$.

(78) Seddon, K. R.; Stark, A.; Torres, M.-J. Influence of chloride, water, and organic solvents on the physical properties of ionic liquids. Pure Appl. Chem. 2000, 72, 2275-2287. 
(79) Kaminsky, M. The concentration and temperature dependence of the viscosity of aqueous solutions of strong electrolytes. III. KCl, $\mathrm{K} 2 \mathrm{SO} 4, \mathrm{MgCl} 2, \mathrm{BeSO} 4$, and $\mathrm{MgSO} 4$ solutions. Z. Phys. Chem. 1957, 12, 206-231.

(80) Doan, T. H.; Sangster, J. Viscosities of concentrated aqueous solutions of some 1: 1, 2: 1, and 3: 1 nitrates at 25. degree. C. J. Chem. Eng. Data 1981, 26, 141-144.

(81) Goldsack, D. E.; Franchetto, R. The viscosity of concentrated electrolyte solutions. I. Concentration dependence at fixed temperature. Can. J. Chem. 1977, 55, 1062-1072.

(82) Breslau, B. R.; Miller, I. F. On the viscosity of concentrated aqueous electrolyte solution. J. Phys. Chem. 1970, 74, 1056-1061.

(83) Hai-Lang, Z.; Shi-Jun, H. Viscosity and Density of Water+ Sodium Chloride. Potassium Chloride Solutions at 298.15 K. J. Chem. Eng. Data 1996, 41, 516-520.

(84) Stoppa, A.; Hunger, J.; Hefter, G.; Buchner, R. Structure and Dynamics of 1-N-Alkyl-3-N-Methylimidazolium Tetrafluoroborate+ Acetonitrile Mixtures. J. Phys. Chem. B 2012, 116, 7509-7521. 